\title{
Nanocomposites of Barium Titanate Nanoparticles Embedded in Thermosetting Polymer Matrices (Novolac Resin/Unsaturated Polyesters/Epoxy Resin): A Comparative Study
}

\author{
Ioannis A. Asimakopoulos ${ }^{1, *(\mathbb{D}}$, Georgios C. Psarras ${ }^{2} \mathbb{C}$ and Loukas Zoumpoulakis ${ }^{1}$ \\ 1 School of Chemical Engineering, Section III “Materials Science and Engineering”, Laboratory Unit \\ "Advanced and Composite Materials", 9-Heroon Polytechniou str., Zografou Campus, National Technical \\ University of Athens, 15773 Athens, Greece; lzoubou@chemeng.ntua.gr \\ 2 Smart Materials \& Nanodielectrics Laboratory, Department of Materials Science, School of Natural Sciences, \\ University of Patras, 26505 Patras, Greece; g.c.psarras@upatras.gr \\ * Correspondence: asimako.ioannis@gmail.com
}

Received: 23 December 2018; Accepted: 28 January 2019; Published: 1 February 2019

\begin{abstract}
Polymer matrix nanocomposites with embedded ferroelectric barium titanate particles were developed and characterized. The utility of such nanocomposites is the energy storage capability that they exhibit, besides their low weight and cost, in comparison to materials that are customarily used for this purpose. The polymers that have been used as matrices in the composites belong to the three most usable thermosetting polymer resins (novolacs, unsaturated polyesters, and epoxy resins), were either laboratory synthesized or commercially supplied. Structure and morphology of the produced composite specimens were studied via Scanning Electron Microscopy (SEM), X-Ray Diffraction (XRD), and Fourier Transformation Infrared Spectroscopy (FTIR). Thermal, mechanical and electrical performance was examined via Differential Scanning Calorimetry (DSC), bending and shear strength tests (three-point method), and Broadband Dielectric Spectroscopy (BDS), respectively. Mechanical shear and bending strength values were determined, as well as mechanical failure mode (brittle or elastomer) were estimated. Dielectric measurements disclosed the presence of four relaxation processes ( $\alpha$-mode, $\beta$-mode, and $\gamma$-mode) and Interfacial Polarization between the system's constituents. The comparative study ended with the calculation of energy density, so that the energy storing capability could be estimated.
\end{abstract}

Keywords: polymer nano-composites; novolac resin; polyester resin; epoxy resin; thermosetting polymerisation; barium titanate; ferroelectric ceramic; mechanical properties; structural characterization; electric properties

\section{Introduction}

A new generation of composite materials has attracted the scientific interest, worldwide. More specifically, the development and study of polymer matrix composite materials with embedded ceramic inclusions, at the nano-scale level, appears to be the focal point of many research efforts [1-3]. These types of materials exhibit a set of attracting electrical properties, which can be exploited in many technological fields and in various uses, such as sensors, actuators, capacitors for storing electric energy, or even as parts of smart materials.

Nowadays, it is well established and accepted that a nano-scaled material has unique properties and is usually quite different and more attractive, in comparison to the same material at the macro-scale. The reason for this is the extensive interface associated with nano-scale and related phenomena. 
The presence of $\mathrm{BaTiO}_{3}$ nano-particles in this type of thermosetting polymer composites, adds to the material's system performance piezoelectric, ferroelectric, and pyroelectric properties, besides its energy storing capability. Additionally, the purpose of using polymers as the matrices of the composite materials is to easily enclose and protect the barium titanate nano-particles from external factors and to create a new integral composite material [1-6].

\section{Materials and Methods}

\subsection{Laboratory Polymer Synthesis}

Novolac and unsaturated polyesters were synthesized in the laboratory. Novolac resin (NV) is a phenol-formaldeyde resin and was created via progressive polymerization. The synthesis of novolac resin, comprises the polycondensation of phenol (Merck), with the presence of formaldehyde (Fluka) and as catalyst acetic acid (Fluka) has been used [2,3,7-11]. Unsaturated polyesters polymerization was held via a polyesterification reaction. In order to synthesize unsaturated polyesters, at least one unsaturated dicarboxylic acid monomer is required. In this research, maleic acid was the unsaturated dicarboxylic acid monomer used. In the synthesis stage of the unsaturated polyesters, not only one, but also a mixture of acids could be used, as long as their molar ratio did not exceed the diol's molar ratio. The saturated diol that has been used was ethylene glycol. These were the raw materials that have been used for the poly-condensation reaction, in which water molecules were produced as a side product. Owing to the fact that water must be continuously removed from the reactor, for the reversible reaction, the distillation method was used. This type of poly-condensation is called poly-esterification, as poly-ester molecules were produced, on account of the poly-condensation of the dicarboxylic acid molecules (maleic acid) and diol molecules (ethylene glycol). The direct poly-esterification reaction was self-catalyzed by the carboxyl groups of the acids components, but due to the reduction in concentration of these groups, as the progress of synthesis reaction carried on with increasing conversation, other catalysts were often employed to maintain the rate of reaction. However, the drawback of using the esterification catalyst is that these components confer a negative effect by colorizing the final product. The theoretical molar proportion of the acid's mixture to diol was 1:1, but practically a molar excess of $10 \%$ of the diol was used, as a result of the diol evaporation, which took place inside the reactor, due to the high temperature (this ratio refers to the feed ratio of the reactor). Toluene was added in the proportion of $3.5 \% \mathrm{v} / \mathrm{w}$ of the total monomers, so that a mixture, with the developed water-an azeotropic mixture-could be collected and removed from the reactor. Hydroquinone was employed as an inhibitor, in the proportion of $0.013 w / w$ of the total amount of monomers. As far as the synthesis stage had finished, unsaturated polyesters were mixed with $30 \% w / w$ styrene (vinylic co-monomer), so that product was ready for the curing process. Four different types of unsaturated polyesters were synthesized, with a difference in the acids' proportions [1,3,12-20]. Their code names are shown in Table 1.

Table 1. The raw materials used for the synthesis of the unsaturated polyesters (feed ratio).

\begin{tabular}{ccccc}
\hline \multirow{2}{*}{$\begin{array}{c}\text { Unsaturated Polyester } \\
\text { Code Name }\end{array}$} & \multicolumn{3}{c}{ \% mol Diacid/Total mol } & \% mol Diole/Total mol \\
\cline { 2 - 5 } & Maleic Acid (M) & Adipic Acid (A) & Phthalic Anydride (PA) & Ethylene Glycol (EG) \\
\hline $\mathrm{M}_{1} \mathrm{~A}_{3} \mathrm{PA}_{6}$ & 10 & 30 & 60 & 110 \\
$\mathrm{M}_{3} \mathrm{~A}_{4} \mathrm{PA}_{3}$ & 30 & 40 & 30 & 110 \\
$\mathrm{M}_{4} \mathrm{~A}_{4} \mathrm{PA}_{2}$ & 40 & 40 & 20 & 110 \\
$\mathrm{M}_{7} \mathrm{~A}_{2} \mathrm{PA}_{1}$ & 70 & 20 & 10 & 110 \\
$\mathrm{M}_{6} \mathrm{~A}_{4}$ & 60 & 40 & 0 & 110 \\
\hline
\end{tabular}

Details about controlling the polymerization process and the calculation processes of the Acid Number and the kinetic parameters (for all of the above polyesters), has been described in previous research work $[1,3,12-16,20]$ and the results are shown in Tables 2 and 3. 
Table 2. The Acid Number of each laboratory synthesized unsaturated polyester.

\begin{tabular}{cc}
\hline Unsaturated Polyester Code Name & A.N. (mg KOH/gr Polyester) \\
\hline $\mathrm{M}_{1} \mathrm{~A}_{3} \mathrm{PA}_{6}$ & 34 \\
$\mathrm{M}_{3} \mathrm{~A}_{4} \mathrm{PA}_{3}$ & 37 \\
$\mathrm{M}_{4} \mathrm{~A}_{4} \mathrm{PA}_{2}$ & 41 \\
$\mathrm{M}_{7} \mathrm{~A}_{2} \mathrm{PA}_{1}$ & 47 \\
$\mathrm{M}_{6} \mathrm{~A}_{4}$ & 43 \\
\hline
\end{tabular}

Table 3. Kinetic parameters of each laboratory synthesized unsaturated polyester.

\begin{tabular}{|c|c|c|c|c|}
\hline \multirow{2}{*}{ Unsaturated Polyester Code Name } & \multicolumn{2}{|c|}{$\mathrm{E}_{\mathbf{a}}$} & \multirow{2}{*}{$\mathrm{k}_{\mathrm{o}}\left(\mathrm{kg} \cdot \mathrm{mol}^{-2} \cdot \min ^{-1}\right)$} & \multirow{2}{*}{$\mathbf{R}^{2}$} \\
\hline & (kcal/mol) & $(\mathrm{kJ} / \mathrm{mol})$ & & \\
\hline $\mathrm{M}_{1} \mathrm{~A}_{3} \mathrm{PA}_{6}$ & 32.19 & 134.69 & $6.53 \times 10^{13}$ & 0.957 \\
\hline $\mathrm{M}_{3} \mathrm{~A}_{4} \mathrm{PA}_{3}$ & 19.87 & 83.14 & $3.60 \times 10^{7}$ & 0.871 \\
\hline $\mathrm{M}_{4} \mathrm{~A}_{4} \mathrm{PA}_{2}$ & 14.45 & 60.44 & $2.27 \times 10^{4}$ & 0.738 \\
\hline $\mathrm{M}_{7} \mathrm{~A}_{2} \mathrm{PA}_{1}$ & 17.60 & 73.66 & $2.19 \times 10^{6}$ & 0.779 \\
\hline $\mathrm{M}_{6} \mathrm{~A}_{4}$ & 26.27 & 110.09 & $2.92 \times 10^{10}$ & 0.986 \\
\hline
\end{tabular}

\subsection{Composite Specimens Preparation-The Curing Process}

All specimens were developed using the compression molding technique. First, special mixtures have been prepared so that cross-linking reactions could take place afterwards. The polymers that were used as the polymer matrices in composites, belong to the phenol-formaldehyde resins (particularly to novolacs), unsaturated polyester resins, as well as epoxy resins. Novolac resin was prepared for curing, after adding the hardener-curing agent hexamethylenotetramine, purchased by Merck, in a ratio of $7: 2 w / w[2,3,11]$. The unsaturated polyesters that have been used are the ones mentioned in Table 2, together with a commercial one (thixotropic polyester with accelerator, Neotex, Athens, Greece) with the code name CUP (commercial unsaturated polyester). The preparation of these mixtures has discussed, in detail, in previous work [1]. As far as the epoxy resin is concerned, a commercial type for electrical purposes has been used (Epoxol 2874, Neotex S.A., Athens, Greece), which has the code name CER (commercial epoxy resin). The curing ratio was $58 \% w / w$ of the epoxy resin's quantity, with commercial hardener. All of the above mixtures were mixed with a specific amount of barium titanate nano-powder (Sigma Aldrich, Taufkirchen, Germany) (with mean diameter in the range: $30-50 \mathrm{~nm})$, in the following pre-selected proportions $(0 \% w / w, 3 \% w / w, 5 \% w / w, 10 \% w / w$, $15 \% w / w, 20 \% w / w \mathrm{BaTiO}_{3}$ ). These final liquid/solid (resin/nano-particles) mixtures were placed into steel moldings, for the compression molding technique. The curing program consisted of the following stages (for the specimens with unsaturated polyesters and epoxy resin: (i) Nano-composites under no pressure, at $60{ }^{\circ} \mathrm{C}$, for $15 \mathrm{~min}$; and (ii) nano-composites under a pressure of $8.5 \mathrm{MPa}$, at $60^{\circ} \mathrm{C}$, for $15 \mathrm{~min}[1,3,12-20]$. While the curing program for the specimens with novolac consisted of: (i) Nano-composites at $140{ }^{\circ} \mathrm{C}$, for one hour, with no pressure applied, and (ii) nano-composites at $170{ }^{\circ} \mathrm{C}$, for one hour, while applying a pressure of $8.5 \mathrm{MPa}$ [2,3,9-11]. Each category of the developed composite specimens had the following code names: NV-BT, $\mathrm{M}_{1} \mathrm{~A}_{3} \mathrm{PA}_{6}-\mathrm{BT}, \mathrm{M}_{3} \mathrm{~A}_{4} \mathrm{PA}_{3}-\mathrm{BT}, \mathrm{M}_{4} \mathrm{~A}_{4} \mathrm{PA}_{2}-\mathrm{BT}$, $\mathrm{M}_{6} \mathrm{~A}_{4}-\mathrm{BT}, \mathrm{M}_{7} \mathrm{~A}_{2} \mathrm{PA}_{1}-\mathrm{BT}, \mathrm{CUP}-\mathrm{BT}$, and CER-BT, combined with barium titanate nano-particles (BT), in the following concentrations: $0 \%, 3 \%, 5 \%, 10 \%, 15 \%$ and $20 \% w / w$.

\subsection{Structural Characterization}

Two basic structural parameters were studied via Scanning Electron Microscopy (SEM): Specimen's surface topography and nanoparticles dispersion, through each polymer matrix. SEM (FEI Quanta 200, FEI Company, Dawson Creek Drive, Hillsboro, USA) was also equipped with Element Dispersive X-Ray Analysis (EDAX) for element identity identification and $\% w / w$ content of each element. Another basic instrument that has been used for the structure examination, is X-Ray Diffraction (XRD). The XRD 
device is a Siemens D5000 Diffractometer (Siemens, Germany), using a Cu Ka source, scanning in the range of $5^{\circ}$ to $120^{\circ}$, with a step of $0.0020^{\circ}$ and a step-time of 1 sec. Structure, and more specifically, the chemical bonds, were also studied via Vibrational Spectroscopy, and particular by means of an Infrared Spectroscopy, via Fourier Transformation (FT-IR) (Perkin Elmer FT-IR Spectrum GM-Waltham, MA, USA.). FT-IR measurements took place in the spectrum region between $4000-200 \mathrm{~cm}^{-1}$ (middle infrared irradiation), in the form of transmittance versus wavelength. All developed specimens were studied via SEM-EDAX, XRD, and FT-IR; the results have been presented in the Results and Discussion part of the study.

\subsection{Thermal Analysis}

Thermal transitions and characteristic important temperatures of the specimens were studied via Differential Scanning Calorimetry (DSC). The employed Differential Scanning Calorimeter is a Q200 (TA Instruments, Lukens Drive, New Castle, PA, USA.), operating at a scan rate of $10^{\circ} \mathrm{C} / \mathrm{min}$. Specimens of all developed samples were placed in an aluminum crucible, while an empty aluminum crucible was served as a reference material. The DSC thermal program consisted of the following stages-temperature was varied from ambient to $200^{\circ} \mathrm{C}$, followed by an isothermal remain at $200^{\circ} \mathrm{C}$, for $1 \mathrm{~min}$. Afterwards, the cooling process consisted of cooling until $0^{\circ} \mathrm{C}$ (via nitrogen flow), again with a cooling rate of $10^{\circ} \mathrm{C} / \mathrm{min}$.

\subsection{Mechanical Characterization}

The Three-Point Method was used for the mechanic tests, in order to test the shear (ASTM-NORM D 2344-65T) and bending (DIN-53452) strength of each specimen [21]. Procedurally, the only difference between these two tests was that, in the shear strength test, the distance between the two points was $10 \mathrm{~mm}$, while in the bending strength test, it was $100 \mathrm{~mm}$. No special conditions were needed for the tests, as the tests took place at room temperature $\left(25^{\circ} \mathrm{C}\right)$. A deflection in the micrometer range was the parameter that was measured in both tests and, afterwards, the shear and bending strength were calculated in MPa [21,22]. Dimensionally, the developed specimens were classified in two categories-specimens that were suitable for the shear tests, with a length of $13.5 \mathrm{~cm}$, width of $17 \mathrm{~mm}$, and a thickness of $3 \mathrm{~mm}$, while the specimens for the bending tests were $13 \mathrm{~cm}$ in length, $10 \mathrm{~mm}$ in width, and $3 \mathrm{~mm}$ in thickness.

\subsection{Dielectric Characterization}

All composite materials manufactured in this project were chosen for their dielectric properties, owing to the barium titanate nano-particles. This is the reason why dielectric characterization is very crucial. Broadband Dielectric Spectroscopy (BDS) was used to examine dielectric properties in the frequency range of $0.1 \mathrm{~Hz}$ to $1 \mathrm{MHz}$, using an Alpha-N Frequency Response Analyser (Novocontrol Technologies, Hundsagen, Germany). The employed dielectric cell (BDS-1200, Novocontrol) was a parallel-plate capacitor, with two gold-plated electrodes. Temperature was controlled via a Novotherm device, also supplied by Novocontrol, with an accuracy of $\pm 0.1^{\circ} \mathrm{C}$. Experimental data were obtained by performing the isothermal frequency scans, for each examined specimens, from $30^{\circ} \mathrm{C}$ to $160{ }^{\circ} \mathrm{C}$, with a temperature step of $10^{\circ} \mathrm{C}$. The amplitude of the applied test voltage was kept constant at $1000 \mathrm{mV}$. Representative specimens from all of the developed polymer matrices were subjected to BDS measurements $\left(0 \%, 5 \%, 10 \%\right.$, and $20 \% w / w$ in the $\mathrm{BaTiO}_{3}$ content).

\section{Results}

\subsection{Scanning Electron Microscopy (SEM) and Energy Dispersive X-Ray Spectroscopy (EDAX)} Characterization

From Figures 1 and 2, the topography of the Au-plated specimen's surface, can be observed. In all of the specimens, the dispersion of the barium titanate nanoparticles is quite satisfactory, as no large 
agglomerates seem to have been created. The shape of a typical barium titanate dispersion within a polymer matrix, looks like a white cloud with a bigger concentration of barium titanate nano-particles in the core of the cloud, in comparison to the more distant places of each cloud. For comparison, a magnitude of 800 times has been selected for one specimen of every polymer matrix category. More details have been given for the specimen with an unsaturated polyester $\mathrm{M}_{7} \mathrm{~A}_{2} \mathrm{PA}_{1}$ matrix, as it is a typical image of all these specimens, with all of their observed characteristics (Figure 1). Figure 2a shows the surface of the specimen with a CER matrix, where there is a clear similarity with a specimen with an NV matrix (subfigure). Table 4 and Figure 2b,c, show the concentration of each element that has been measured via the Energy Dispersive X-Ray Spectroscopy (EDAX), through spot characterization, inside a typical $\mathrm{BaTiO}_{3}$ cloud, for the three selected nano-composites specimens shown in Figures 1 and 2a. It is obvious that carbon, barium, oxygen, and titanium were detected, as expected. The highest proportion of all detected chemical elements corresponded to carbon, due to the polymer matrix.

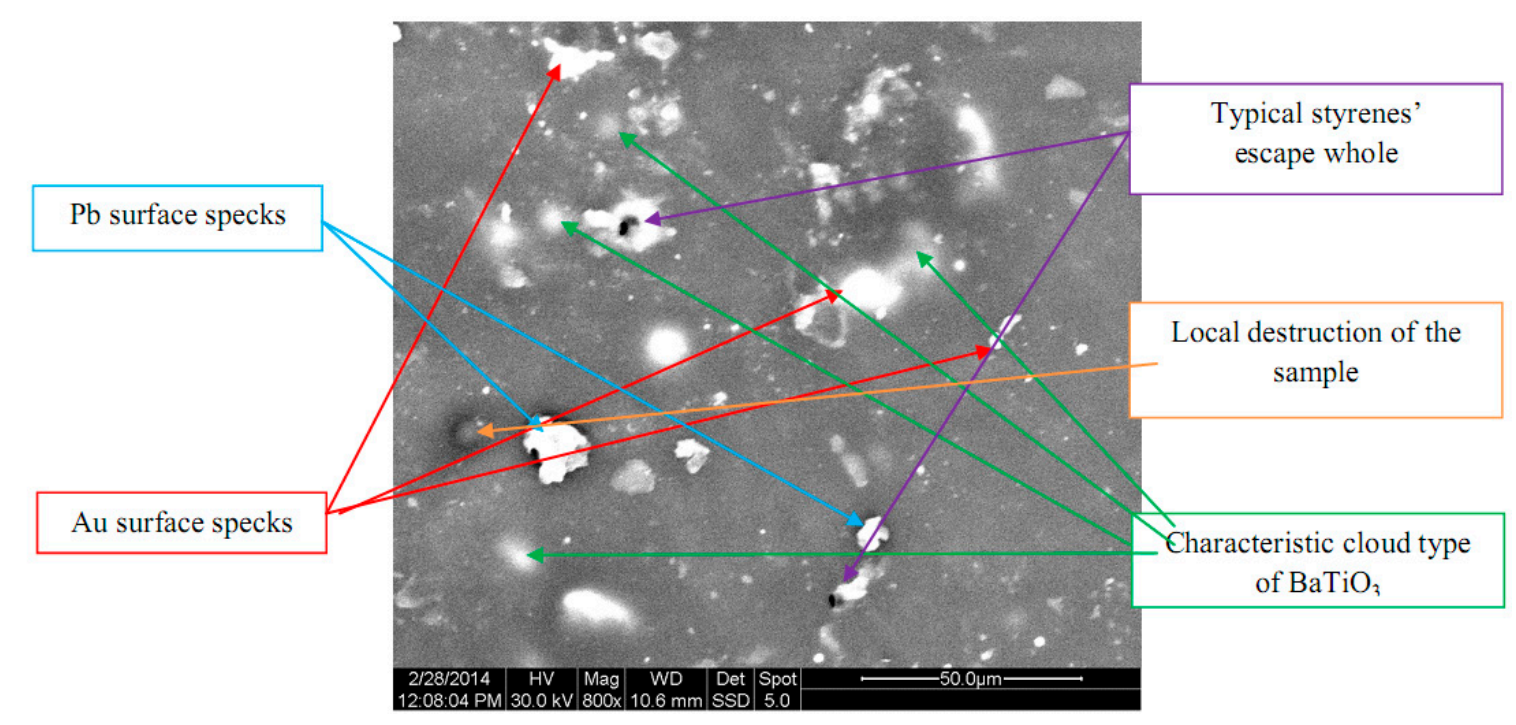

Figure 1. Scanning Electron Microscopy (SEM) image of the specimen with an $\mathrm{M}_{7} \mathrm{~A}_{2} \mathrm{PA}_{1}$ polymer matrix and $20 \% w / w \mathrm{BaTiO}_{3}$, at a magnification of 800 times $(\times 800)$.

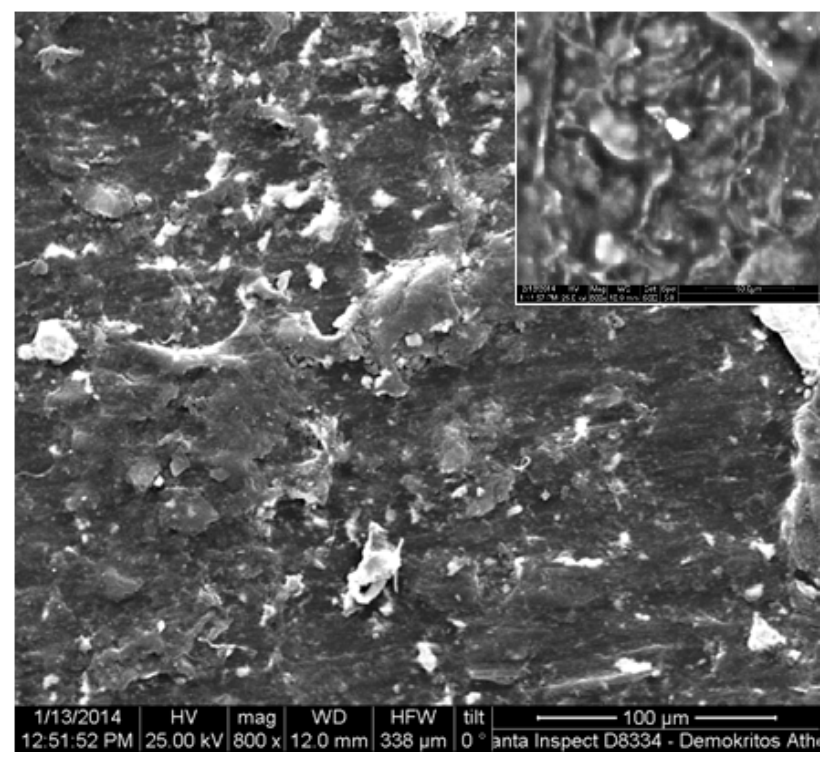

(a)

Figure 2. Cont. 


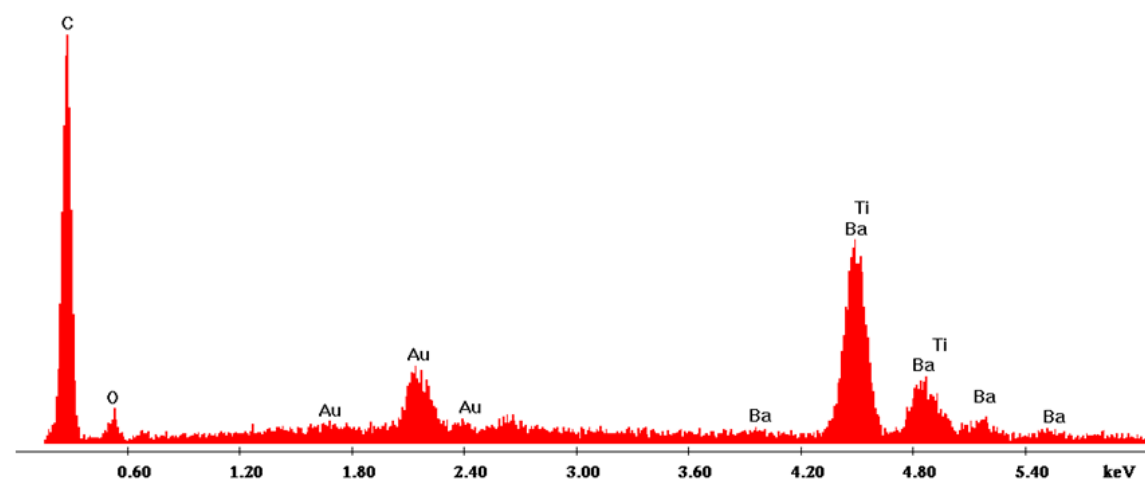

(b)

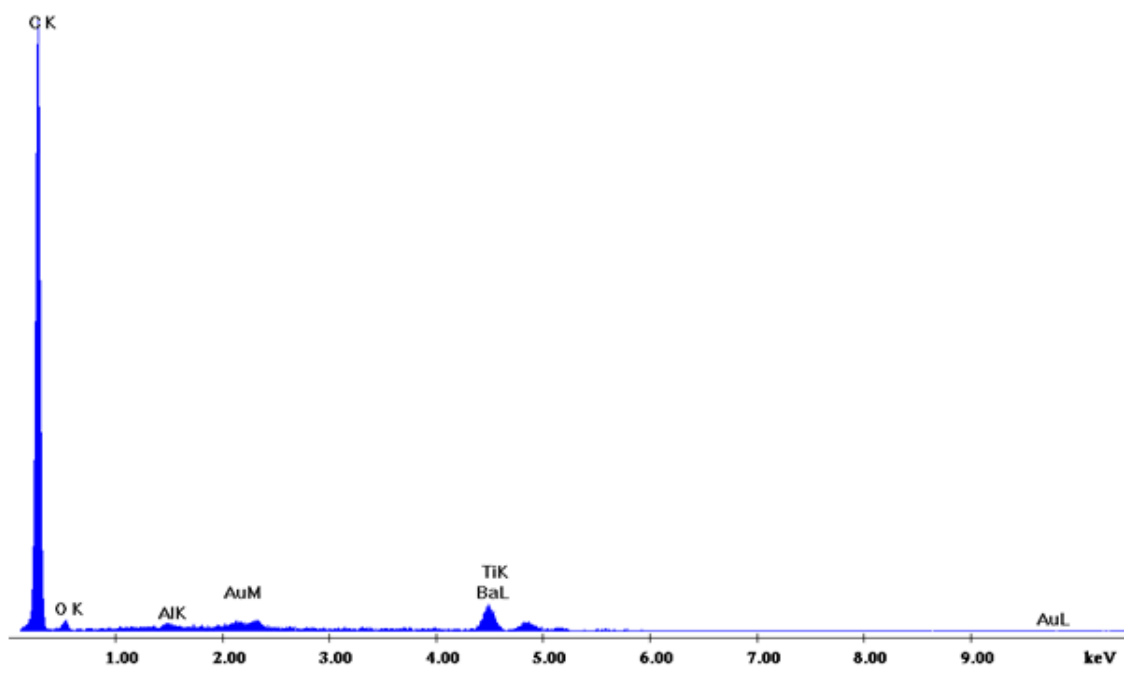

(c)

Figure 2. (a) Scanning Electron Microscopy (SEM) image of the specimen with commercial epoxy resin (CER) polymer matrix and $20 \% w / w \mathrm{BaTiO}_{3}$, at a magnification of 800 times $(\times 800)$ (subfigure: Scanning Electron Microscopy (SEM) image of the specimen with a novolac resin (NV) polymer matrix and $20 \% w / w \mathrm{BaTiO}_{3}$, at a magnification of 800 times $(\times 800)$ ). (b) Energy Dispersive X-Ray Spectroscopy (EDAX) measurement for the $20 \% w / w \mathrm{BaTiO}_{3}$ specimen, with a CER polymer matrix, and (c) an Energy Dispersive X-Ray Spectroscopy (EDAX) measurement for the $20 \% w / w \mathrm{BaTiO}_{3}$ specimen, with an NV polymer matrix.

Table 4. X-ray spectroscopy measurements from the three selected specimens in Figures 1 and 2.

\begin{tabular}{cccccc}
\hline \multirow{2}{*}{ Specimen Type } & \multicolumn{5}{c}{ Chemical Element \% w/w } \\
\cline { 2 - 6 } & $\mathbf{C ~ ( K )}$ & O (K) & Ba (L) & Ti (K) & Total \\
\hline $20 \% w / w$ NV-BT & 86.73 & 5.44 & 6.35 & 1.49 & 100.00 \\
$20 \% w / w \mathrm{M}_{7} \mathrm{~A}_{2} \mathrm{PA}_{1}$-BT & 43.27 & 16.79 & 33.42 & 6,52 & 100.00 \\
$20 \% w / w \mathrm{CER}-\mathrm{BT}$ & 65.57 & 5.00 & 23.67 & 5.76 & 100.00 \\
\hline
\end{tabular}

\subsection{X-Ray Diffraction (XRD) Characterization}

Figure 3 depicts the XRD spectra of composites with $0 \%, 5 \%, 20 \%$, and $100 \% w / w$ in the $\mathrm{BaTiO}_{3}$ and $\mathrm{M}_{1} \mathrm{~A}_{3} \mathrm{PA}_{6}$ unsaturated polyester polymer matrix. The graphs are a mixture of an amorphous and a crystal material, where each phase prevails over the other, compared to the observed intensity (counts) $[1-3,11,12,17-20]$. The crystal phase is due to the barium titanate's crystallinity, and the amorphous phase is due to the amorphous phase of each polymer matrix. The crystal phase appears with sharp peaks in the XRD spectra, while the amorphous phase appears with a wide peak in the low 
$2 \theta$ values. All specimens have exhibited exactly the same behavior, so a similar XRD spectra, as the ones shown in Figure 3. Table 5, shows the $2 \theta$ values of all developed nano-composites, which are related to each polymer amorphous wide peak. It is obvious that the amorphous peak of all laboratory unsaturated polyesters are located approximately at $21^{\circ}$, while at about $20^{\circ}$, for the commercial one. Whereas, the novolac's and the epoxy's amorphous peak was located at about $18^{\circ}$.

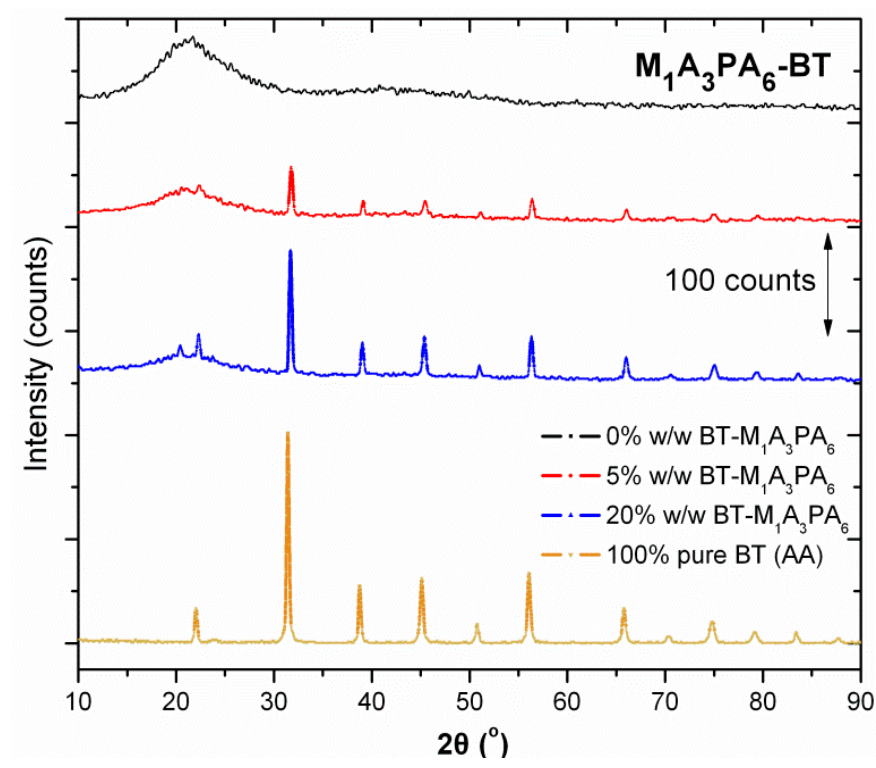

Figure 3. X-Ray Diffraction spectra from the pure $\mathrm{BaTiO}_{3}$ nano-particles and specimens with $0 \%, 5 \%$, and $20 \% w / w$ in $\mathrm{BaTiO}_{3}$, in the $\mathrm{M}_{1} \mathrm{~A}_{3} \mathrm{PA}_{6}$ unsaturated polyester polymer matrix.

Table 5. $2 \theta$ values from each amorphous polymer peak of all developed nano-composites.

\begin{tabular}{cc}
\hline Composite Polymer Matrix & $\left.\mathbf{2 \theta} \mathbf{(}^{\circ}\right)$ \\
\hline $\mathrm{M}_{1} \mathrm{~A}_{3} \mathrm{PA}_{6}$ & 21.20 \\
$\mathrm{M}_{3} \mathrm{~A}_{4} \mathrm{PA}_{3}$ & 21.37 \\
$\mathrm{M}_{4} \mathrm{~A}_{4} \mathrm{PA}_{2}$ & 21.50 \\
$\mathrm{M}_{6} \mathrm{~A}_{4}$ & 21.40 \\
$\mathrm{M}_{7} \mathrm{~A}_{2} \mathrm{PA}_{1}$ & 21.49 \\
$\mathrm{CUP}$ & 19.72 \\
$\mathrm{NV}$ & 18.38 \\
$\mathrm{CER}$ & 17.67 \\
\hline
\end{tabular}

\subsection{Infra Red Spectroscopy via Fourier Transformation (FT-IR) Characterization}

Figure 4 is a classical FT-IR spectra of the nano-composites consisting of barium titanate nano-particles embedded in polymer matrix. Specifically, Figure 4 depicts composites with the $\mathrm{M}_{3} \mathrm{~A}_{4} \mathrm{PA}_{3}$ unsaturated polyester as polymer matrix for all developed specimens from $0 \% w / w$ to $20 \% w / w$, in $\mathrm{BaTiO}_{3}$, the same procedure occurred for all developed composite categories. The window of values, at the left graph, ranged from $4000 \mathrm{~cm}^{-1}$ to $200 \mathrm{~cm}^{-1}$, while the right one was a focused spectrum from $1800 \mathrm{~cm}^{-1}$ to $350 \mathrm{~cm}^{-1}$, so that all characteristic peaks could be more easily distinguishable. All formed peaks have been identified and classified, throughout, in Tables $6-8$, for all developed specimens. The symbols used for the identification of each peak, can be explained as follows: (i) Regarding the shape of the peak-(sh) means sharp and (br) means broad; (ii) regarding the magnitude of the peak-(s) means strong, (m) means medium, (w) means weak and (pl) means plateau. The most important, characteristic wavelength bands for each nano-composite category have been shaded in purple so that they are easily distinguishable. For the nano-composites with the novolac resin as a polymer matrix, the most important wavelength bands were-stretch vibration of the phenol's - $\mathrm{OH}$ group, stretch vibration within the aromatic carbon ring of the phenol, bending vibration of the phenol's $-\mathrm{OH}$ group, and vibrations within the hardener 
hexamethylenetetramine (HMTA) molecule (Table 6). For the nano-composites with the unsaturated polyester resin as a polymer matrix, the most important wavelength bands were-stretch vibration of the ester bond, the vibration of the alcohol bond, and the hardener styrene stretch vibration (Table 7). Finally, the nano-composites with the epoxy resin as a polymer matrix the most important wavelength bands were-bisphenol (BPA) vibration, vibration of the hardener isophoronodiamine (IPD or IPDA) molecule, vibration of the ether bond, and of the oxirane group [1,3,13-16,23-29].
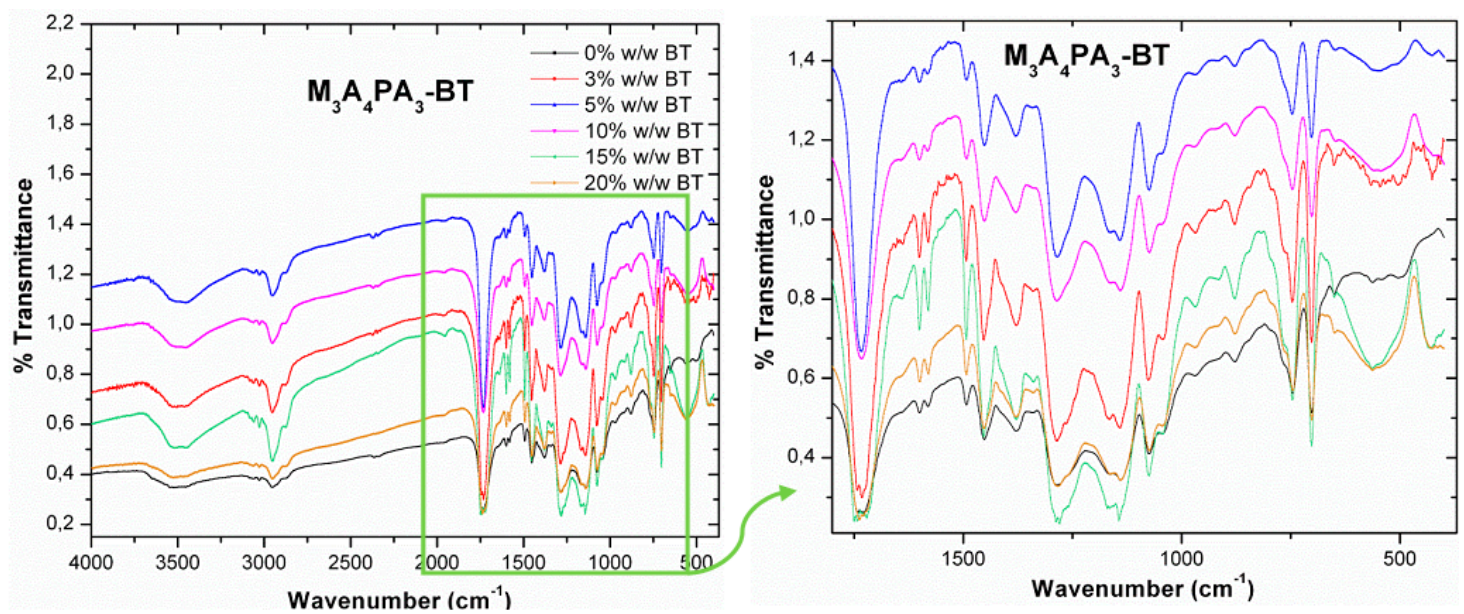

Figure 4. Infrared Spectroscopy via Fourier Transformation (FT-IR) spectra from specimens with 0-20\% content in $\mathrm{BaTiO}_{3}$ nano-particles in the $\mathrm{M}_{3} \mathrm{~A}_{4} \mathrm{PA}_{3}$, at a wavelength range between: $4000-200 \mathrm{~cm}^{-1}$ (left) and $1800-350 \mathrm{~cm}^{-1}$ (right).

Table 6. FT-IR spectra characteristic peaks from the novolac resin (NV).

\begin{tabular}{|c|c|c|}
\hline \multirow{2}{*}{ Characteristic Chemical Group } & \multirow{2}{*}{$\begin{array}{l}\text { Wavelength from } \\
\text { Literature }\left(\mathrm{cm}^{-1}\right)\end{array}$} & \multirow{2}{*}{$\begin{array}{c}\text { Peak Appearance Wavelength }\left(\mathrm{cm}^{-1}\right) \\
\text { Cured Novolac Resin (NV) } \\
\text { (Phenol-Phormaldehyde resin) }\end{array}$} \\
\hline & & \\
\hline -OH stretch vibration & $3435-3383$ & $3367 \mathrm{w} / \mathrm{br}$ \\
\hline $\mathrm{CH},>\mathrm{CH}_{2},-\mathrm{CH}_{3}$ stretch vibration, aliphatic parts & $2960-2850$ & $2954 \mathrm{w} / \mathrm{sh}, 2923 \mathrm{~m} / \mathrm{sh}, 2856 \mathrm{~m} / \mathrm{br}$ \\
\hline $\mathrm{C}=\mathrm{C}$ stretch vibration within the aromatic ring & $1635-1630$ & $1637 \mathrm{~s} / \mathrm{br}$ \\
\hline $\mathrm{C}=\mathrm{C}$ stretch vibration within the aromatic ring & $1615-1600$ & $1610 \mathrm{~s} / \mathrm{br}$ \\
\hline $\mathrm{C}=\mathrm{C}$ stretch vibration within the aromatic ring & $1510-1504$ & $1509 \mathrm{w} / \mathrm{sh}, 1498 \mathrm{w} / \mathrm{sh}$ \\
\hline $\begin{array}{c}\mathrm{CH},>\mathrm{CH}_{2},-\mathrm{CH}_{3} \text { bending vibration aliphatic } \\
\text { parts (asymmetric bending) }\end{array}$ & $1470-1457$ & $1473 \mathrm{w} / \mathrm{sh}, 1457 \mathrm{~m} / \mathrm{sh}$ \\
\hline -OH bending vibration & $1438-1415$ & $1438 \mathrm{w} / \mathrm{sh}$ \\
\hline $\begin{array}{c}\mathrm{CH},>\mathrm{CH}_{2},-\mathrm{CH}_{3} \text { bending vibration aliphatic } \\
\text { parts (symmetric bending) }\end{array}$ & $1378-1370$ & $1371 \mathrm{~m} / \mathrm{sh}$ \\
\hline$-\mathrm{OH}$ bending vibration & $1330-1320$ & $1340 \mathrm{w} / \mathrm{br}$ \\
\hline $\mathrm{CH}$, bending Hexa (HMTA) & $1244-1240$ & $1238 \mathrm{~s} / \mathrm{sh}$ \\
\hline stretch vibration within the aromatic ring & $1210-1100$ & $1213 \mathrm{w} / \mathrm{br}, 1141 \mathrm{w} / \mathrm{br}$ \\
\hline Aromatic ring asymmetric rings & $1100-1109$ & $1100 \mathrm{w} / \mathrm{sh}$ \\
\hline Aromatic ring asymmetric rings & $1097-1050$ & $1072 \mathrm{w} / \mathrm{br}, 1047 \mathrm{w} / \mathrm{br}$ \\
\hline Hexa (HMTA) & $1007-1000$ & $1008 \mathrm{~s} / \mathrm{sh}$ \\
\hline $\begin{array}{c}\text { C-H Deformation vibration } 1 \text { or } 2 \text { neighbour } \mathrm{H} \text { of } \\
\text { benzene ring (para-substituents) }\end{array}$ & $814-810$ & $813 \mathrm{~m} / \mathrm{sh}$ \\
\hline $\begin{array}{l}\text { C-H Deformation vibration } 3 \text { or } 4 \text { neighbour H of } \\
\text { benzene ring (meta- or ortho-substituents) }\end{array}$ & $778-750$ & $757 \mathrm{~m} / \mathrm{br}$ \\
\hline $\begin{array}{c}\mathrm{C}-\mathrm{H} \text { Deformation vibration } 5 \text { neighbour } \mathrm{H} \text { of } \\
\text { benzene ring }\end{array}$ & $672-666$ & $673 \mathrm{~m} / \mathrm{sh}$ \\
\hline
\end{tabular}


Table 7. FT-IR spectra characteristic peaks from unsaturated polyesters $\left(\mathrm{M}_{1} \mathrm{~A}_{3} \mathrm{PA}_{6}, \mathrm{M}_{3} \mathrm{~A}_{4} \mathrm{PA}_{3}\right.$, $\mathrm{M}_{4} \mathrm{~A}_{4} \mathrm{PA}_{2}, \mathrm{M}_{6} \mathrm{~A}_{4}, \mathrm{M}_{7} \mathrm{~A}_{2} \mathrm{PA}_{1}$, and CUP).

\begin{tabular}{|c|c|c|c|c|c|c|c|}
\hline \multirow{3}{*}{$\begin{array}{l}\text { Characteristic } \\
\text { Chemical Group }\end{array}$} & \multirow{3}{*}{$\begin{array}{l}\text { Wavelength from } \\
\text { Literature }\left(\mathrm{cm}^{-1}\right)\end{array}$} & \multicolumn{6}{|c|}{ Peak Appearance Wavelength $\left(\mathrm{cm}^{-1}\right)$} \\
\hline & & \multicolumn{6}{|c|}{ Composite's Cured Unsaturated Polyester Polymer Matrix } \\
\hline & & $\mathbf{M}_{1} \mathbf{A}_{3} \mathbf{P A}_{6}$ & $\mathbf{M}_{3} \mathbf{A}_{4} \mathbf{P A}_{3}$ & $\mathbf{M}_{4} \mathbf{A}_{4} \mathbf{P A}_{2}$ & $\mathbf{M}_{6} \mathbf{A}_{4}$ & $\mathbf{M}_{7} \mathbf{A}_{2} \mathbf{P A}_{1}$ & CUP \\
\hline $\begin{array}{l}\text {-OH stretch } \\
\text { vibration }\end{array}$ & $3600-3200$ & $\begin{array}{l}3512 \mathrm{~m} / \mathrm{br} \\
3452 \mathrm{~m} / \mathrm{br}\end{array}$ & $\begin{array}{l}3523 \mathrm{~m} / \mathrm{br} \\
3452 \mathrm{~m} / \mathrm{br}\end{array}$ & $3446 \mathrm{~s} / \mathrm{br}$ & $\begin{array}{l}3540 \mathrm{w} / \mathrm{br} \\
3459 \mathrm{w} / \mathrm{br}\end{array}$ & $3456 \mathrm{~s} / \mathrm{br}$ & $\begin{array}{l}3534 \mathrm{w} / \mathrm{br} \\
3443 \mathrm{w} / \mathrm{br}\end{array}$ \\
\hline $\begin{array}{l}\text { C-H stretch } \\
\text { vibration, styrene's } \\
\text { aromatic ring }\end{array}$ & 3024 & $\begin{array}{l}3061 \mathrm{w} / \mathrm{sh} \\
3026 \mathrm{w} / \mathrm{sh}\end{array}$ & $\begin{array}{l}3064 \mathrm{w} / \mathrm{sh} \\
3028 \mathrm{w} / \mathrm{sh}\end{array}$ & $\begin{array}{l}3064 \mathrm{w} / \mathrm{sh} \\
3030 \mathrm{w} / \mathrm{sh}\end{array}$ & $\begin{array}{l}3061 \mathrm{w} / \mathrm{sh} \\
3029 \mathrm{w} / \mathrm{sh}\end{array}$ & $\begin{array}{l}3062 \mathrm{w} / \mathrm{sh} \\
3026 \mathrm{w} / \mathrm{sh}\end{array}$ & $\begin{array}{l}3062 \mathrm{w} / \mathrm{sh} \\
3028 \mathrm{w} / \mathrm{sh}\end{array}$ \\
\hline $\begin{array}{c}>\mathrm{CH}_{2},-\mathrm{CH}_{3} \text { stretch } \\
\text { vibration, aliphatic } \\
\text { parts }\end{array}$ & $2980-2950$ & $2953 \mathrm{~m} / \mathrm{sh}$ & $2954 \mathrm{~m} / \mathrm{sh}$ & $2956 \mathrm{~m} / \mathrm{sh}$ & $2958 \mathrm{~m} / \mathrm{sh}$ & $2951 \mathrm{~m} / \mathrm{sh}$ & $2933 \mathrm{~m} / \mathrm{sh}$ \\
\hline $\begin{array}{c}>\mathrm{CH}_{2} \text { vibration } \\
\text { stretch, circular and } \\
\text { linear parts }\end{array}$ & $2890-2850$ & $2885 \mathrm{w} / \mathrm{sh}$ & $2875 \mathrm{w} / \mathrm{sh}$ & $2881 \mathrm{w} / \mathrm{sh}$ & $2878 \mathrm{w} / \mathrm{sh}$ & $2883 \mathrm{w} / \mathrm{sh}$ & $\begin{array}{l}28857 \\
\mathrm{w} / \mathrm{sh}\end{array}$ \\
\hline $\mathrm{CO}_{2}$ & 2360 & $2345 \mathrm{w} / \mathrm{sh}$ & $\begin{array}{l}2362 \mathrm{w} / \mathrm{sh} \\
2339 \mathrm{w} / \mathrm{br}\end{array}$ & $\begin{array}{l}2364 \mathrm{~m} / \mathrm{sh} \\
2337 \mathrm{w} / \mathrm{sh}\end{array}$ & $2351 \mathrm{w} / \mathrm{sh}$ & $2343 \mathrm{w} / \mathrm{sh}$ & $2346 \mathrm{w} / \mathrm{sh}$ \\
\hline $\begin{array}{l}>\mathrm{C}=\mathrm{O} \text { Ester bond } \\
\text { stretch vibration }\end{array}$ & $1736-1726$ & $1734 \mathrm{~s} / \mathrm{sh}$ & $1728 \mathrm{~s} / \mathrm{sh}$ & $1734 \mathrm{~s} / \mathrm{sh}$ & $1736 \mathrm{~s} / \mathrm{sh}$ & $1734 \mathrm{~s} / \mathrm{sh}$ & $1731 \mathrm{~s} / \mathrm{sh}$ \\
\hline $\begin{array}{l}\mathrm{C}=\mathrm{C} \text { stretch } \\
\text { vibration within the } \\
\text { aromatic ring }\end{array}$ & $\begin{array}{l}1600, \\
1580 \\
1500\end{array}$ & $\begin{array}{l}1598 \mathrm{~m} / \mathrm{sh}, \\
1579 \mathrm{~m} / \mathrm{sh}, \\
1492 \mathrm{~m} / \mathrm{sh}\end{array}$ & $\begin{array}{l}1598 \mathrm{w} / \mathrm{sh}, \\
1579 \mathrm{w} / \mathrm{sh}, \\
1492 \mathrm{w} / \mathrm{sh}\end{array}$ & $\begin{array}{l}1602 \mathrm{w} / \mathrm{br}, \\
1583 \mathrm{w} / \mathrm{sh}, \\
1492 \mathrm{w} / \mathrm{sh}\end{array}$ & $\begin{array}{c}1603 \mathrm{w} / \mathrm{sh}, \\
-, \\
1495 \mathrm{w} / \mathrm{sh}\end{array}$ & $\begin{array}{c}-, \\
-, \\
1490 \mathrm{w} / \mathrm{sh}\end{array}$ & $\begin{array}{l}1600 \mathrm{~m} / \mathrm{sh}, \\
1581 \mathrm{~m} / \mathrm{sh}, \\
1494 \mathrm{~m} / \mathrm{sh}\end{array}$ \\
\hline $\begin{array}{l}\text { C-C stretch } \\
\text { vibration within the } \\
\text { aromatic ring }\end{array}$ & $1480-1400$ & $1450 \mathrm{~m} / \mathrm{sh}$ & $1452 \mathrm{~s} / \mathrm{sh}$ & $1452 \mathrm{~m} / \mathrm{sh}$ & $1456 \mathrm{~m} / \mathrm{sh}$ & $1450 \mathrm{~m} / \mathrm{sh}$ & $1453 \mathrm{~s} / \mathrm{sh}$ \\
\hline $\begin{array}{l}\mathrm{CH}_{3} \text { Symmetric } \\
\text { bending vibration }\end{array}$ & $1390-1370$ & $1373 \mathrm{~m} / \mathrm{sh}$ & $1379 \mathrm{~m} / \mathrm{sh}$ & $1386 \mathrm{~m} / \mathrm{sh}$ & $1393 \mathrm{~m} / \mathrm{sh}$ & $1394 \mathrm{~m} / \mathrm{sh}$ & $1381 \mathrm{w} / \mathrm{sh}$ \\
\hline $\mathrm{C}_{\mathrm{sp} 3}-\mathrm{O}$ (ester bond) & $1330-1200$ & $1284 \mathrm{~s} / \mathrm{sh}$ & $1284 \mathrm{~s} / \mathrm{sh}$ & $1286 \mathrm{~s} / \mathrm{sh}$ & $1250 \mathrm{w} / \mathrm{br}$ & $1282 \mathrm{~s} / \mathrm{sh}$ & $1283 \mathrm{~s} / \mathrm{sh}$ \\
\hline $\begin{array}{l}\mathrm{C}_{\mathrm{sp} 3}-\mathrm{O} \text { (alcohol } \\
\text { bond) }\end{array}$ & $1200-1100$ & $\begin{array}{l}1166 \mathrm{w} / \mathrm{sh} \\
1136 \mathrm{~m} / \mathrm{sh}\end{array}$ & $\begin{array}{l}1163 \mathrm{w} / \mathrm{sh}, \\
1139 \mathrm{~m} / \mathrm{sh}\end{array}$ & $1155 \mathrm{~m} / \mathrm{br}$ & $1162 \mathrm{~m} / \mathrm{sh}$ & $1161 \mathrm{~m} / \mathrm{sh}$ & $1122 \mathrm{~m} / \mathrm{sh}$ \\
\hline $\begin{array}{l}\mathrm{C}-\mathrm{H} \text { «in plane» } \\
\text { vibrations }\end{array}$ & 1080-1036 & $1072 \mathrm{~m} / \mathrm{sh}$ & $1074 \mathrm{~m} / \mathrm{sh}$ & $1074 \mathrm{~m} / \mathrm{sh}$ & $1065 \mathrm{~m} / \mathrm{br}$ & $1074 \mathrm{~m} / \mathrm{sh}$ & $1068 \mathrm{~m} / \mathrm{sh}$ \\
\hline $\begin{array}{c}\mathrm{C}=\mathrm{C} \text { unsaturated } \\
\text { polyester's double } \\
\text { bond }\end{array}$ & 982 & $964 \mathrm{w} / \mathrm{sh}$ & $970 \mathrm{w} / \mathrm{br}$ & - & $978 \mathrm{w} / \mathrm{sh}$ & $981 \mathrm{w} / \mathrm{br}$ & $990 \mathrm{w} / \mathrm{br}$ \\
\hline $\begin{array}{l}C=C \text { Styrene's } \\
\text { double bond }\end{array}$ & 912 & $904 \mathrm{w} / \mathrm{sh}$ & - & $925 \mathrm{w} / \mathrm{br}$ & - & - & $913 \mathrm{w} / \mathrm{br}$ \\
\hline $\begin{array}{l}\text { C-H Deformation } \\
\text { vibration «out of } \\
\text { plane» } 1 \text { or } 2 \\
\text { neighbour } \mathrm{H} \text { of } \\
\text { benzene ring }\end{array}$ & $876-815$ & $877 \mathrm{w} / \mathrm{sh}$ & $877 \mathrm{w} / \mathrm{sh}$ & $879 \mathrm{w} / \mathrm{sh}$ & $\begin{array}{l}877 \mathrm{w} / \mathrm{sh} \\
814 \mathrm{w} / \mathrm{br}\end{array}$ & $879 \mathrm{w} / \mathrm{sh}$ & $847 \mathrm{~m} / \mathrm{br}$ \\
\hline $\begin{array}{l}\text { C-H Deformation } \\
\text { vibration «out of } \\
\text { plane» } 3 \text { or } 4 \\
\text { neighbour } \mathrm{H} \text { of } \\
\text { benzene ring }\end{array}$ & 750 & $744 \mathrm{~s} / \mathrm{sh}$ & $746 \mathrm{~s} / \mathrm{sh}$ & $748 \mathrm{~s} / \mathrm{sh}$ & $764 \mathrm{~m} / \mathrm{sh}$ & $754 \mathrm{~s} / \mathrm{sh}$ & $745 \mathrm{~s} / \mathrm{sh}$ \\
\hline $\begin{array}{l}\text { C-H Deformation } \\
\text { vibration «out of } \\
\text { plane» } 5 \text { neighbour } \\
\text { H of benzene ring }\end{array}$ & 700,666 & $\begin{array}{l}700 \mathrm{~s} / \mathrm{sh} \\
650 \mathrm{w} / \mathrm{sh}\end{array}$ & $702 \mathrm{~s} / \mathrm{sh}$ & $\begin{array}{l}702 \mathrm{~s} / \mathrm{sh} \\
650 \mathrm{w} / \mathrm{br}\end{array}$ & $703 \mathrm{~s} / \mathrm{sh}$ & $702 \mathrm{~s} / \mathrm{sh}$ & $\begin{array}{l}700 \mathrm{~s} / \mathrm{sh} \\
649 \mathrm{w} / \mathrm{br}\end{array}$ \\
\hline
\end{tabular}


Table 8. FT-IR spectra characteristic peaks from the epoxy resin (the CER).

\begin{tabular}{|c|c|c|}
\hline \multirow{2}{*}{ Characteristic Chemical Group } & \multirow{2}{*}{$\begin{array}{l}\text { Wavelength from } \\
\text { Literature }\left(\mathrm{cm}^{-1}\right)\end{array}$} & \multirow{2}{*}{$\begin{array}{c}\text { Peak Appearance Wavelength }\left(\mathrm{cm}^{-1}\right) \\
\text { Cured Epoxy Resin (CER) }\end{array}$} \\
\hline & & \\
\hline -OH stretch vibration & $4000-3400$ & $3416 \mathrm{~s} / \mathrm{br}$ \\
\hline $\mathrm{C}-\mathrm{H}$ Aromatic ring stretch vibration, & 3024 & $3034 \mathrm{w} / \mathrm{sh}$ \\
\hline$>\mathrm{CH}_{2},-\mathrm{CH}_{3}$ stretch vibration, aliphatic parts & $\begin{array}{l}3100-2800 \text { (usually } \\
\text { between } 2960-2850 \text { ) }\end{array}$ & $2923 \mathrm{~s} / \mathrm{sh}, 2854 \mathrm{w} / \mathrm{sh}$ \\
\hline $\mathrm{CO}_{2}$ & 2360 & $2360 \mathrm{~m} / \mathrm{sh}$ \\
\hline $\mathrm{C}_{\mathrm{sp} 3}-\mathrm{O}$ alcohol BPA stretch vibration & $1753-1743$ & $1750 \mathrm{w} / \mathrm{br}$ \\
\hline $\begin{array}{c}\text { Aromatic ring (benzene's backbone vibration, } \\
\text { BPA molecule) }\end{array}$ & $1610-1605$ & $1608 \mathrm{~s} / \mathrm{sh}$ \\
\hline Isophorono-Diamine (IPD) & 1580 & $1582 \mathrm{w} / \mathrm{sh}$ \\
\hline $\mathrm{C}=\mathrm{C}$ vibration stretch within the aromatic ring & $1510-1505$ & $1509 \mathrm{~s} / \mathrm{sh}$ \\
\hline $\begin{array}{l}>\mathrm{CH}_{2},-\mathrm{CH}_{3} \text { aliphatic parts bending vibration } \\
\text { («in plane» deformation vibration) }\end{array}$ & $1450 \pm 20$ & $1456 \mathrm{~m} / \mathrm{sh}$ \\
\hline -OH bending vibration & $1410-1260$ & $1384 \mathrm{w} / \mathrm{sh}, 1362 \mathrm{w} / \mathrm{sh}, 1297 \mathrm{w} / \mathrm{sh}$ \\
\hline $\mathrm{C}_{\mathrm{sp} 3}-\mathrm{O}$ stretch vibration, (aromatic ether) & $1330-1200$ & $1247 \mathrm{~s} / \mathrm{sh}$ \\
\hline stretch vibration, aromatic ring & 1180 & $1181 \mathrm{~s} / \mathrm{sh}$ \\
\hline $\mathrm{C}_{\mathrm{sp} 3}-\mathrm{OH}$ Alcohol stretch vibration, & $1150-1050$ & $1109 \mathrm{~m} / \mathrm{sh}, 1083 \mathrm{w} / \mathrm{sh}$ \\
\hline $\begin{array}{l}\text { aromatic } C \text { trans formations of ether bond } \\
(\text { C-O-C) stretch vibration }\end{array}$ & $1039-1034$ & $1037 \mathrm{~s} / \mathrm{sh}$ \\
\hline $\begin{array}{l}\text { C-O-C Epoxy group stretch vibration } \\
\text { (oxirane bond) }\end{array}$ & 930-914 & $957 \mathrm{w} / \mathrm{br}, 934 \mathrm{w} / \mathrm{br}$ \\
\hline $\begin{array}{l}\text { C-H Deformation vibration «out of plane» } \\
\text { individual } \mathrm{H} \text { within aromatic ring }\end{array}$ & 876 & $874 \mathrm{w} / \mathrm{br}$ \\
\hline C-H Aromatic ring bending (out of plane) & $830-829$ & $828 \mathrm{~s} / \mathrm{sh}$ \\
\hline $\begin{array}{c}\text { C-H Deformation vibration «out of plane» } 3 \text { or } 4 \\
\text { neighbour } \mathrm{H} \text { of aromatic ring }\end{array}$ & 750 & $749 \mathrm{~m} / \mathrm{br}, 734 \mathrm{~m} / \mathrm{br}$ \\
\hline C-H Deformation vibration «out of plane» & 675 & $698 \mathrm{~m} / \mathrm{sh}$ \\
\hline $\begin{array}{c}\text { C-H Deformation vibration «out of plane» } 5 \\
\text { neighbour H of aromatic ring }\end{array}$ & 666,640 & $668 \mathrm{w} / \mathrm{sh}$ \\
\hline
\end{tabular}

Barium titanate has five characteristic regions in which peaks rise. These five characteristic regions have been commented on, in detail, in previous work [1]. These five characteristic peaks of barium titanate appear in all of the nano-composites examined with FT-IR spectroscopy; the results together, with the comments, have been listed in Table 9. A difference was easily noticeable in the characteristics of each peak. This difference was attributed to the increase of the proportion of $\mathrm{BaTiO}_{3}$ in the specimens, thus, a variation in the intensity of each peak occurred. As a conclusion about all examined FT-IR spectra, the following remarks could be made-there was a characteristic region which was attributed to the presence of each polymer matrix and there was a number of characteristic peaks which were attributed to the presence of the barium titanate nano-particles. 
Table 9. FT-IR spectra characteristic peaks from all of the developed nano-composites.

\begin{tabular}{|c|c|c|c|c|c|c|c|c|c|}
\hline \multirow{3}{*}{$\begin{array}{l}\text { Characteristic } \\
\text { Chemical Group }\end{array}$} & \multirow{3}{*}{$\begin{array}{l}\text { Wavelength from } \\
\text { Literature }\left(\mathrm{cm}^{-1}\right)\end{array}$} & \multicolumn{8}{|c|}{ Peak Appearance Wavelength $\left(\mathrm{cm}^{-1}\right)$} \\
\hline & & \multicolumn{8}{|c|}{ Composite Category } \\
\hline & & NV-BT & $\mathrm{M}_{1} \mathrm{~A}_{3} \mathrm{PA}_{6}-\mathrm{BT}$ & $\mathrm{M}_{3} \mathrm{~A}_{4} \mathrm{PA}_{3}-\mathrm{BT}$ & $\mathrm{M}_{4} \mathrm{~A}_{4} \mathrm{PA}_{2}-\mathrm{BT}$ & $\mathrm{M}_{6} \mathrm{~A}_{4}-\mathrm{BT}$ & $\mathrm{M}_{7} \mathbf{A}_{2} \mathbf{P A}_{1}-\mathrm{BT}$ & CUP-BT & CER-BT \\
\hline $\begin{array}{c}\mathrm{Ba}-\mathrm{O}, \mathrm{Ti}-\mathrm{O} \\
\text { stretch vibration }\end{array}$ & $\begin{array}{c}450-405 \mathrm{~m} / \mathrm{sh} \\
\text { (region ii) }\end{array}$ & $\begin{array}{l}422 \mathrm{~m} / \mathrm{br}(3 \%) \\
428 \mathrm{~m} / \mathrm{br}(5 \%) \\
420 \mathrm{~s} / \mathrm{br}(10 \%) \\
431 \mathrm{~s} / \mathrm{br}(15 \%) \\
426 \mathrm{~s} / \mathrm{br}(20 \%)\end{array}$ & $\begin{array}{c}400 \mathrm{~s} / \mathrm{br} \\
(3 \%-20 \%)\end{array}$ & $\begin{array}{c}426 \mathrm{~m} / \mathrm{br} \\
(3 \%, 5 \%) \\
424 \mathrm{~s} / \mathrm{br}(10 \%) \\
430 \mathrm{~s} / \mathrm{sh}(15 \%) \\
426 \mathrm{~s} / \mathrm{sh}(20 \%)\end{array}$ & $\begin{array}{l}\text { very weak }(3 \%) \\
443 \mathrm{w} / \mathrm{br}(5 \%) \\
428 \mathrm{~s} / \mathrm{br}(10 \%) \\
432 \mathrm{~s} / \mathrm{br}(15 \%) \\
432 \mathrm{~s} / \mathrm{sh}(20 \%)\end{array}$ & $\begin{array}{l}432 \mathrm{~s} / \mathrm{br} \\
(3 \%, 5 \%) \\
428 \mathrm{~s} / \mathrm{br} \\
(10-20 \%)\end{array}$ & $\begin{array}{c}447 \mathrm{~m} / \mathrm{sh}(3 \%) \\
439 \mathrm{w} / \mathrm{br}(5 \%) \\
439 \mathrm{~m} / \mathrm{br}(10 \%) \\
405 \mathrm{~s} / \mathrm{br}(15 \%) \\
430 \mathrm{~s} / \mathrm{br}(20 \%)\end{array}$ & $\begin{array}{c}458 \mathrm{~m} / \mathrm{br} \\
(3 \%, 5 \%) \\
431 \mathrm{~s} / \mathrm{br} \\
(10 \%-20 \%)\end{array}$ & $\begin{array}{c}425 \mathrm{~s} / \mathrm{br}(3 \%) \\
433 \mathrm{~s} / \mathrm{br}(5 \%) \\
429 \mathrm{~s} / \mathrm{br}(10 \%) \\
432 \mathrm{~s} / \mathrm{br}(15 \%) \\
416 \mathrm{~s} / \mathrm{br}(20 \%)\end{array}$ \\
\hline $\begin{array}{c}\text { Ti-O } \\
\text { stretch vibration }\end{array}$ & $\begin{array}{l}700-530 \mathrm{~s} / \mathrm{sh} \\
(\text { region i) }\end{array}$ & $\begin{array}{c}698 \mathrm{w} / \mathrm{br}(3 \%) \\
700 \mathrm{~s} / \mathrm{br} \\
(5 \%-15 \%) \\
698 \mathrm{~s} / \mathrm{br}(20 \%)\end{array}$ & $\begin{array}{c}540 \mathrm{~s} / \mathrm{br} \\
(3 \%-20 \%)\end{array}$ & $\begin{array}{c}545 \mathrm{~m} / \mathrm{br}(3 \%) \\
547 \mathrm{~m} / \mathrm{br}(5 \%) \\
543 \mathrm{~m} / \mathrm{br}(10 \%) \\
565 \mathrm{~s} / \mathrm{br}(15 \%) \\
563 \mathrm{~s} / \mathrm{br}(20 \%)\end{array}$ & $\begin{array}{c}\text { very weak (3\%) } \\
563 \mathrm{~s} / \mathrm{br}(5 \%) \\
559 \mathrm{~s} / \mathrm{br}(10 \%) \\
553 \mathrm{~s} / \mathrm{br}(15 \%) \\
561 \mathrm{~s} / \mathrm{br}(20 \%)\end{array}$ & $\begin{array}{l}504 \mathrm{~s} / \mathrm{sh} \\
(3 \%, 5 \%) \\
504 \mathrm{~s} / \mathrm{br} \\
(10-20 \%)\end{array}$ & $\begin{array}{c}514 \mathrm{~s} / \mathrm{br}(3 \%) \\
518 \mathrm{~s} / \mathrm{br}(5 \%) \\
540 \mathrm{~s} / \mathrm{br}(10 \%) \\
547 \mathrm{~s} / \mathrm{br}(15 \%) \\
545 \mathrm{~s} / \mathrm{br}(20 \%)\end{array}$ & $\begin{array}{c}541 \mathrm{~s} / \mathrm{br} \\
(3 \%-20 \%)\end{array}$ & $\begin{array}{c}558 \mathrm{~s} / \mathrm{br}(3 \%) \\
561 \mathrm{~s} / \mathrm{br}(5 \%) \\
560 \mathrm{~s} / \mathrm{br}(10 \%) \\
559 \mathrm{~s} / \mathrm{br} \\
(15 \%, 20 \%)\end{array}$ \\
\hline $\begin{array}{l}\mathrm{Ba}-\mathrm{O}, \mathrm{Ti}-\mathrm{O} \\
\text { stretch vibration }\end{array}$ & $\begin{array}{c}860-852 \mathrm{w} / \mathrm{sh} \\
\text { (region iv) }\end{array}$ & $\begin{array}{l}\text { Overlap with } \\
\text { NV peak: } \\
874 \mathrm{w} / \mathrm{br}\end{array}$ & $\begin{array}{c}\text { Overlap with } \\
\mathrm{M}_{1} \mathrm{~A}_{3} \mathrm{PA}_{6} \text { peak: } \\
877 \mathrm{w} / \mathrm{sh}\end{array}$ & $\begin{array}{c}\text { Overlap with } \\
\mathrm{M}_{3} \mathrm{~A}_{4} \mathrm{PA}_{3} \text { peak: } \\
877 \mathrm{w} / \mathrm{sh}\end{array}$ & $\begin{array}{c}\text { Overlap with } \\
\mathrm{M}_{4} \mathrm{~A}_{4} \mathrm{PA}_{2} \text { peak: } \\
879 \mathrm{w} / \mathrm{sh}\end{array}$ & $\begin{array}{c}\text { Overlap with } \\
\mathrm{M}_{6} \mathrm{~A}_{4} \text { peak: } \\
877 \mathrm{w} / \mathrm{sh}\end{array}$ & $\begin{array}{c}\text { Overlap with } \\
\mathrm{M}_{7} \mathrm{~A}_{2} \mathrm{PA}_{1} \text { peak: } \\
879 \mathrm{w} / \mathrm{sh}\end{array}$ & $\begin{array}{l}\text { Overlap with } \\
\text { CUP peak: } \\
847 \mathrm{~m} / \mathrm{br}\end{array}$ & $\begin{array}{l}\text { Overlap with } \\
\text { CER peak: } \\
874 \mathrm{w} / \mathrm{br}\end{array}$ \\
\hline $\begin{array}{c}\mathrm{C}-\mathrm{O} \\
\text { stretch vibration }\end{array}$ & $\begin{array}{l}1444-1440 \mathrm{~m} / \mathrm{sh} \\
\text { (region iii) }\end{array}$ & - & $\begin{array}{c}\text { Overlap with } \\
\mathrm{M}_{1} \mathrm{~A}_{3} \mathrm{PA}_{6} \text { peak: } \\
1450 \mathrm{~m} / \mathrm{sh}\end{array}$ & $\begin{array}{c}\text { Overlap with } \\
\mathrm{M}_{3} \mathrm{~A}_{4} \mathrm{PA}_{3} \text { peak: } \\
1452 \mathrm{~s} / \mathrm{sh}\end{array}$ & $\begin{array}{c}\text { Overlap with } \\
\mathrm{M}_{4} \mathrm{~A}_{4} \mathrm{PA}_{2} \text { peak: } \\
1452 \mathrm{~m} / \mathrm{sh}\end{array}$ & $\begin{array}{c}\text { Overlap with } \\
\mathrm{M}_{6} \mathrm{~A}_{4} \text { peak: } \\
1456 \mathrm{~m} / \mathrm{sh}\end{array}$ & $\begin{array}{c}\text { Overlap with } \\
\mathrm{M}_{7} \mathrm{~A}_{2} \mathrm{PA}_{1} \text { peak: } \\
1450 \mathrm{~m} / \mathrm{sh}\end{array}$ & $\begin{array}{l}\text { Overlap with } \\
\text { CUP peak: } \\
1453 \text { s/sh }\end{array}$ & $\begin{array}{l}\text { Overlap with } \\
\text { CER peak: } \\
1456 \mathrm{~m} / \mathrm{sh}\end{array}$ \\
\hline $\begin{array}{c}-\mathrm{OH} \\
\text { stretch vibration }\end{array}$ & $\begin{array}{c}3433-3389 \mathrm{~m} / \mathrm{br} \\
(\text { region v) }\end{array}$ & $\begin{array}{l}\text { Overlap with } \\
\text { NV peak: } \\
3416 \mathrm{~s} / \mathrm{br}\end{array}$ & $\begin{array}{c}\text { Overlap with } \\
\mathrm{M}_{1} \mathrm{~A}_{3} \mathrm{PA}_{6} \text { peak: } \\
3452 \mathrm{~m} / \mathrm{br}\end{array}$ & $\begin{array}{l}\text { Overlap with } \\
\mathrm{M}_{3} \mathrm{~A}_{4} \mathrm{PA}_{3} \text { peak: } \\
3452 \mathrm{~m} / \mathrm{br}\end{array}$ & $\begin{array}{c}\text { Overlap with } \\
\mathrm{M}_{4} \mathrm{~A}_{4} \mathrm{PA}_{2} \text { peak: } \\
3446 \mathrm{~s} / \mathrm{br}\end{array}$ & $\begin{array}{c}\text { Overlap with } \\
\mathrm{M}_{6} \mathrm{~A}_{4} \text { peak: } \\
3459 \mathrm{w} / \mathrm{br}\end{array}$ & $\begin{array}{c}\text { Overlap with } \\
\mathrm{M}_{7} \mathrm{~A}_{2} \mathrm{PA}_{1} \text { peak: } \\
3456 \mathrm{~s} / \mathrm{br}\end{array}$ & $\begin{array}{l}\text { Overlap with } \\
\text { CUP peak: } \\
3443 \mathrm{w} / \mathrm{br}\end{array}$ & $\begin{array}{l}\text { Overlap with } \\
\text { CER peak: } \\
3416 \mathrm{~s} / \mathrm{br}\end{array}$ \\
\hline
\end{tabular}




\subsection{Thermal Characterization}

Thermal Analysis and, specifically, Differential Scanning Calorimetry (DSC) is a method which allows the determination of a number of important characteristic temperatures. The most important of all these characteristic temperatures is the glass transition temperature (Tg), due to the fact that these composites have polymers as matrices. The determination of the $\mathrm{Tg}$ was carried out via the point of inflexion of the transition. Even if all of the polymers employed for the developed composites were the thermosetting ones, practically, an amount of the initial thermoplastic (before the curing took place) never turned to thermosetting, via the curing process. Due to this, $\mathrm{Tg}$ must be calculated and estimated before using such materials. A comparative graph has been depicted in Figure 5, for all of the developed nano-composite categories, except for the nano-composites with the novolac resin as matrix. This happened because the novolac is the most perfectly cured thermosetting polymer, so no thermoplastic region existed after the curing process. By examining the graph, two kinds of behaviors could be detected. In the largest variety of the nano-composites $\left(\mathrm{M}_{1} \mathrm{~A}_{3} \mathrm{PA}_{6}-\mathrm{BT}, \mathrm{M}_{3} \mathrm{~A}_{4} \mathrm{PA}_{3}-\mathrm{BT}\right.$, $\mathrm{M}_{4} \mathrm{~A}_{4} \mathrm{PA}_{2}-\mathrm{BT}, \mathrm{M}_{7} \mathrm{~A}_{2} \mathrm{PA}_{1}-\mathrm{BT}$, and CER-BT), Tg seemed to increase, while the content of the specimen in the barium titanate also increased. On the other hand, two kinds of nano-composites $\left(\mathrm{M}_{6} \mathrm{~A}_{4}-\mathrm{BT}\right.$ and CUP-BT) exhibited exactly a different behavior, so the Tg decreased while the content of the specimen in the barium titanate, increased. This behavior occurs, sometimes, in similar polymer matrix composites. In the first case, the barium titanate nano-particles seemed to bind the thermal energy, and did not release it easily, so the specimens could withstand higher temperatures, without harming the remaining thermoplastic regions. As a result, these specimens had higher glass transition temperatures. Contrariwise, the other two composite categories exhibited exactly the opposite behavior, the nano-particles seemed not to bind the thermal energy, so the polymer matrix was affected by a decrease in its Tg. Conclusively, the barium titanate nano-particles, in one case, acted protectively for the polymer matrix, and non-protectively, in the other.

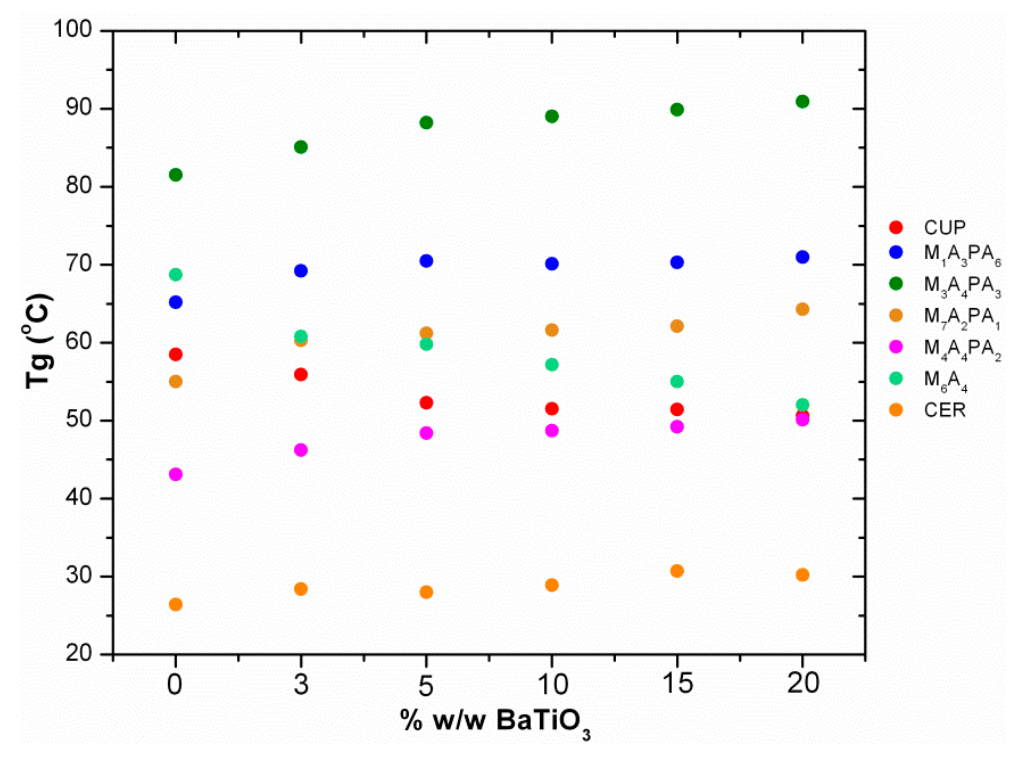

Figure 5. Glass transition temperature ( $\mathrm{Tg}$ ) for all of the developed nano-composite specimens.

\subsection{Mechanical Characterization}

Shear and bending tests took place via the three-point method, in order to calculate the shear and bending strengths of all developed specimens. In Figures 6 and 7, not all composite categories existed. This happened because some of the developed specimens did not fail, in certain tests. For example, the unsaturated polyester with the code name $\mathrm{M}_{1} \mathrm{~A}_{3} \mathrm{PA}_{6}$, displayed a completely elastomeric behavior, so none of the $\mathrm{M}_{1} \mathrm{~A}_{3} \mathrm{PA}_{6}$-BT composites failed in the above-mentioned mechanical tests. In the shear test, all the other developed composites failed, except for the $\mathrm{M}_{1} \mathrm{~A}_{3} \mathrm{PA}_{6}-\mathrm{BT}$ composites. On the contrary, in the 
bending test, none of the composites with a laboratory synthesized unsaturated polyester as a polymer matrix, failed, as they exhibit an almost elastomeric behavior, while the NV-BT, CUP-BT, and the CER-BT composites exhibited a brittle behavior, so the composities of all these categories failed in the bending test. A very important remark was that, by increasing the content of the barium titanate, in all of the developed composites, both for shear and the bending tests, the shear and the bending strength decreased. This fact had to do with the type of adhesion between the nano-particles and the macro-molecular polymer chains, so this meant that by increasing the content of the barium titanates, it became more and more difficult for the nano-particles to disperse between the 3D-cured polymer network. Another very important point was that, the bending strength values exhibited three times higher values in relation to the shear ones, separately for each category. Finally, comparing the mechanical behavior of the three composite categories, the highest strength values were shown by the CER-BT composites, as was expected, followed by the strength values of the composites with the unsaturated polyester polymer matrix, and in the end, the poorest mechanical strength values were displayed by the NV-BT composites.

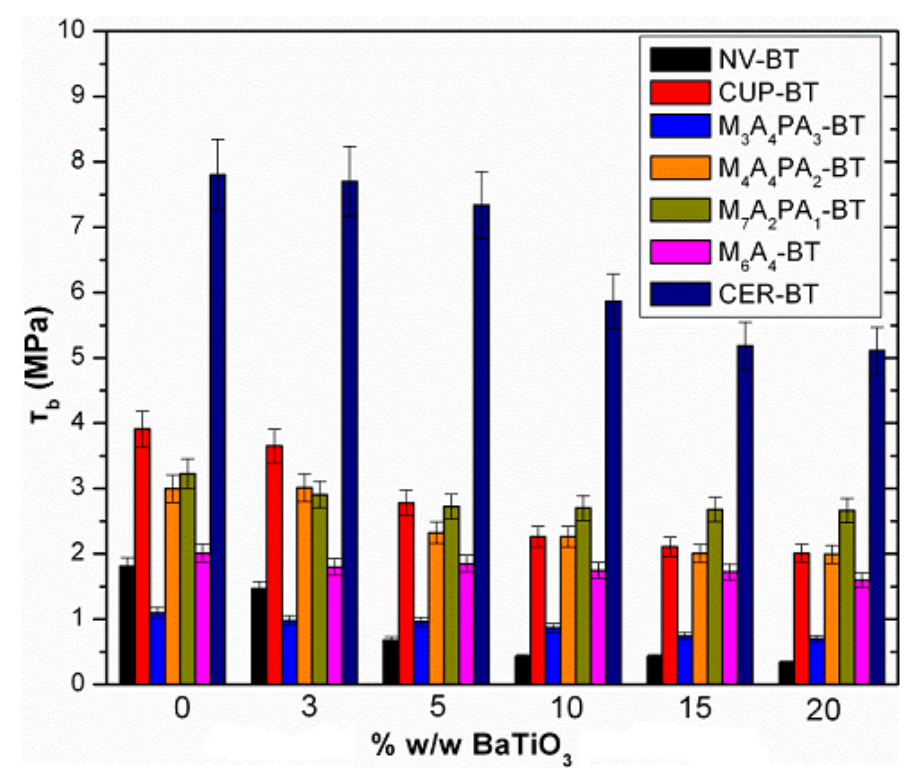

Figure 6. Shear strength values for some of the developed nano-composite specimens.

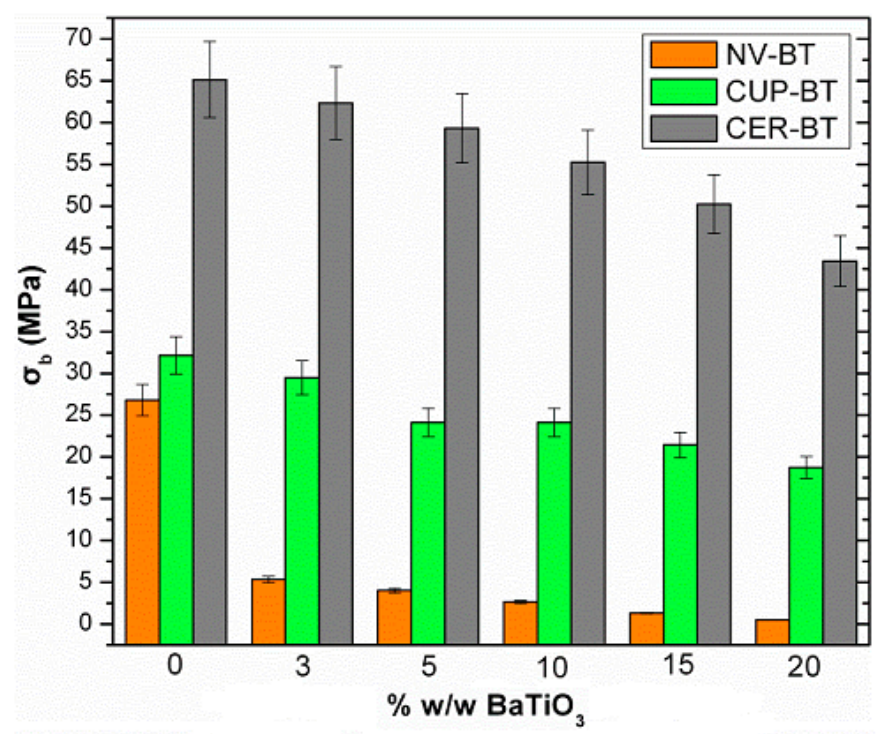

Figure 7. Bending strength values for some of the developed nano-composite specimens. 


\subsection{Broadband Dielectric Spectroscopy (BDS) Characterization}

Dielectric measurements were conducted by means of a broadband dielectric spectroscopy (BDS), in the frequency range of $0.1 \mathrm{~Hz}$ to $10 \mathrm{MHz}$, using an Alpha-N Frequency Response Analyser (Novocontrol Technologies, Hundsagen, Germany). The employed dielectric cell (BDS-1200, Novocontrol) was a parallel-plate capacitor with two gold-plated electrodes (Figure 8). Temperature was controlled via the Novotherm device, supplied also by Novocontrol. Experimental data were obtained by performing isothermal frequency scans, for each examined specimen, from ambient to $150{ }^{\circ} \mathrm{C}$, with a temperature step of $5{ }^{\circ} \mathrm{C}$. The amplitude of the applied test voltage was kept constant at $1000 \mathrm{mV}[2,3]$.
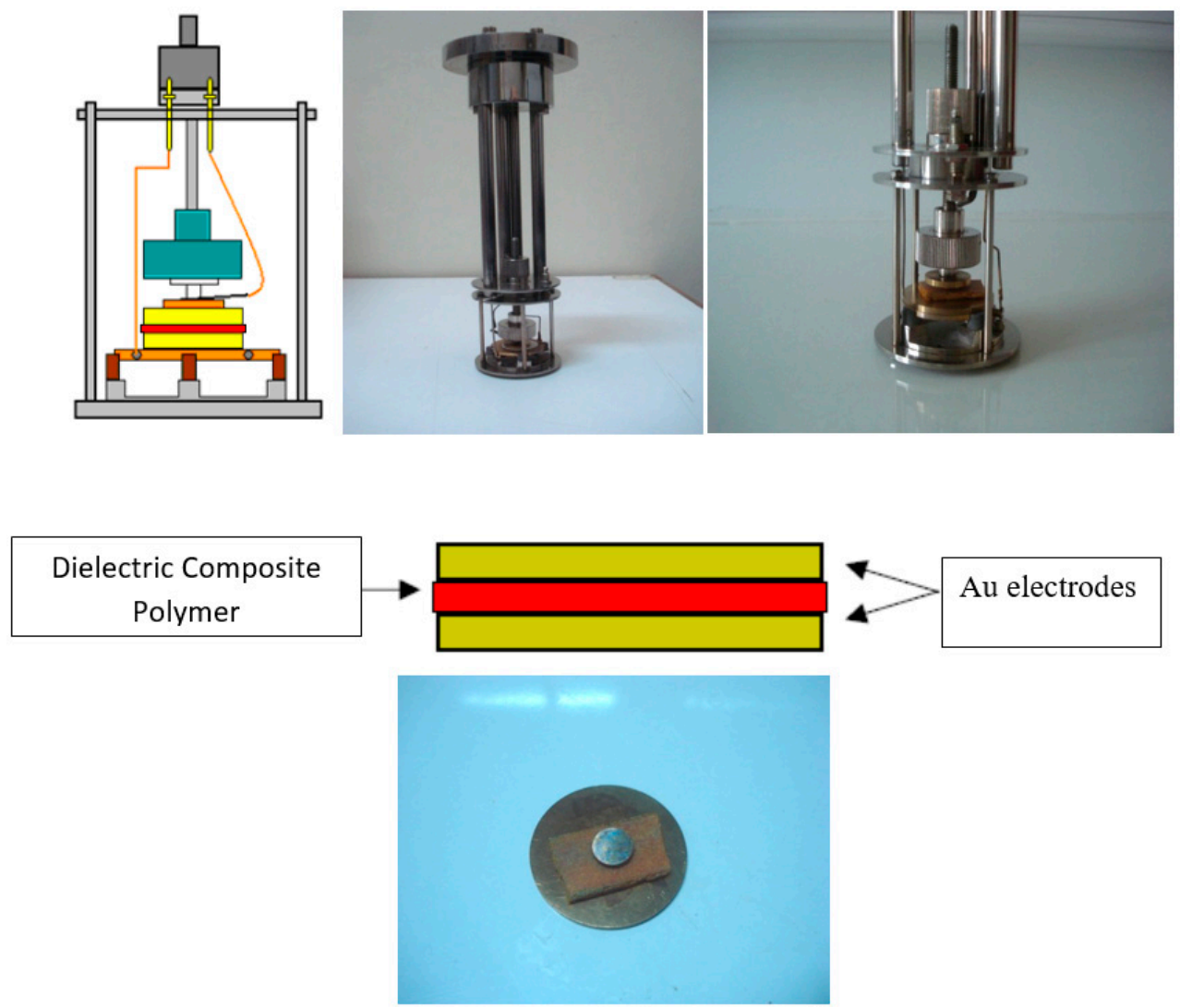

Figure 8. The Broadband Dielectric Spectroscopy (BDS) measurement cell. There were two gold tablets on the top and at the bottom of the layout, and between the two tablets each specimen was placed [2].

Dielectric measurements concerning the developed composites, disclosed the presence of three relaxation processes, which were attributed to the re-arrangement of the polar side groups ( $\beta$-mode), glass-to-rubber transition of the polymer matrix ( $\alpha$-mode), and the Interfacial Polarization between the system's constituents. First, dielectric permittivity was calculated and then, through Equation (1), conversed into the electric modulus. In all cases, the real part of electric permittivity diminished with the frequency reflecting the decrease of polarization, due to the inertia of the dipoles, to follow the alternation of the applied field. The electric modulus is considered as an effective formalism for the investigation of dielectric data, since it eliminates the parasitic and undesirable effect of electrode polarization [1-3,30-32]. Electric modulus is defined as the inverse quantity of complex permittivity, as shown in Equation (1), where $\varepsilon^{\prime}, \mathrm{M}^{\prime}$ and $\varepsilon^{\prime \prime}, \mathrm{M}^{\prime \prime}$ are the real and the imaginary parts of the dielectric permittivity and the electric modulus, respectively.

$$
M^{*}=\frac{1}{\varepsilon^{*}}=\frac{1}{\varepsilon^{\prime}-j \varepsilon^{\prime \prime}}=\frac{\varepsilon^{\prime}}{\mathcal{\varepsilon}^{\prime 2}+\varepsilon^{\prime \prime 2}}+j \frac{\varepsilon^{\prime \prime}}{{\mathcal{E}^{\prime}}^{2}+\varepsilon^{\prime \prime 2}}=M^{\prime}+j M^{\prime \prime}
$$


These relaxation processes can be easily be detected from Figures 9-11, from the graphs of the real part of the dielectric permittivity and the imaginary part of the electric modulus, versus either frequency or temperature. The specimens, with the following specific content in the barium titanate, were not randomly selected, but they were the ones with the highest energy storing capability of each composite category (as will be explained afterwards). So, the three dimensions plots in Figure 9 depict the real part of the dielectric permittivity and the imaginary part of the electric modulus, versus the frequency and the temperature. It was clear that Interfacial Polarization appeared in relatively lower frequencies and relatively higher temperatures than the $\alpha$-relaxation (Figures 9-11), as the Interfacial Polarization's dipoles were bigger than those of the $\alpha$-relaxation, so larger amounts of energy were required. In higher frequency and lower temperature values, $\beta$-relaxation appeared. $\beta$-relaxation was related to a re-arrangement of the polar side groups, so these dipoles were smaller, compared to the larger parts of the polymer chain ( $\alpha$-relaxation), and because of this, a lower level of energy was needed. The cause of the $\beta$-relaxation for the novolac and the epoxy-based composites, was attributed to the re-arrangement of the hydroxyl polar side groups $(-\mathrm{OH})$. Concerning the composites with the unsaturated polyesters as polymer matrix, $\beta$-relaxation was attributed to the re-arrangement of the oxygen molecules, connected with the main polymer chain, by a double bond.
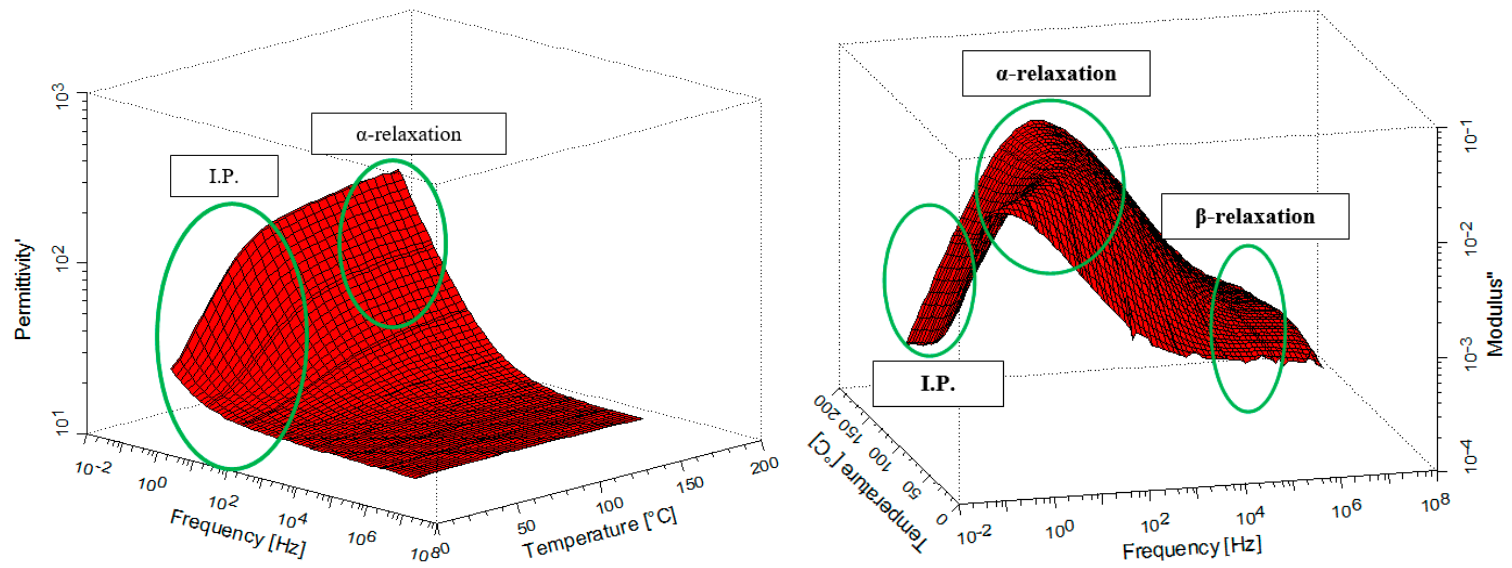

Figure 9. The real part of the dielectric permittivity (left) and the imaginary part of the electric modulus (right), versus frequency and temperature, for the $10 \% w / w \mathrm{BaTiO}_{3}-\mathrm{NV}$ nano- composites.
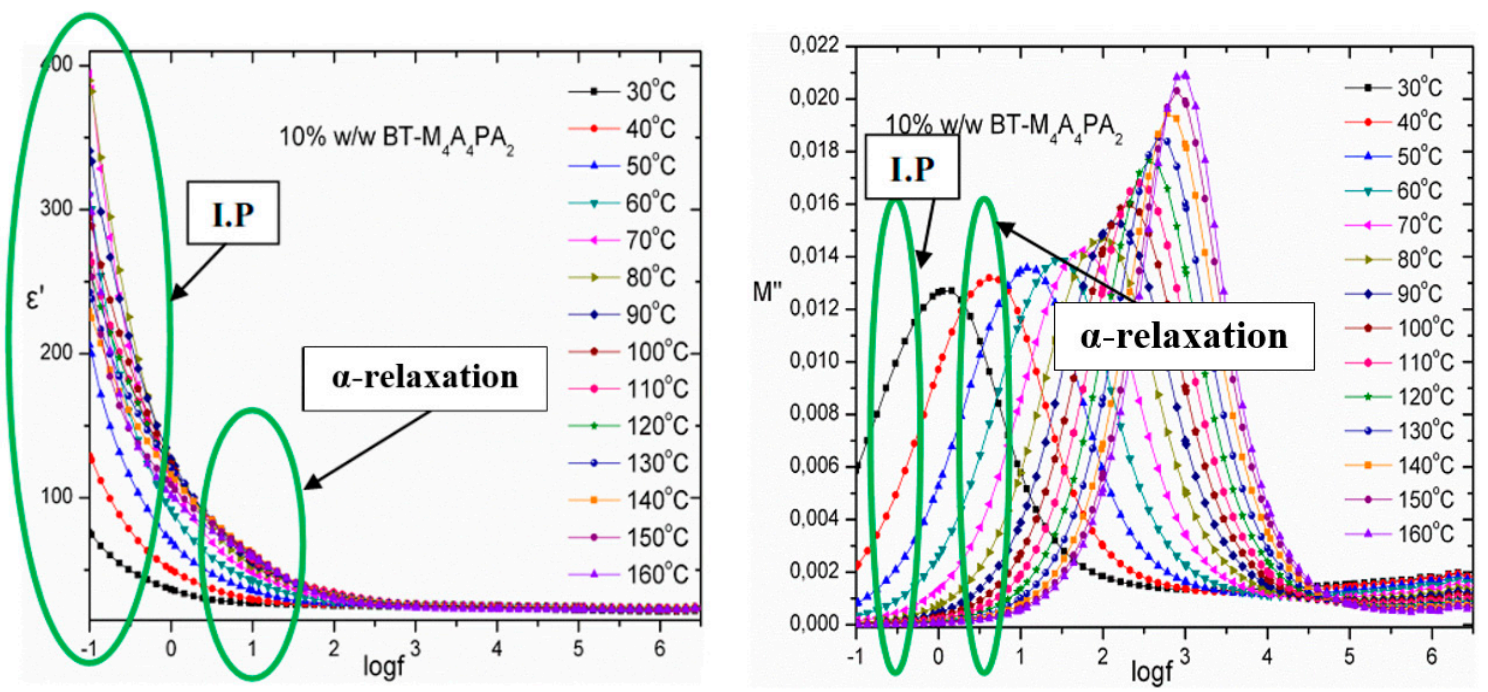

Figure 10. The real part of the dielectric permittivity (left) and the imaginary part of the electric modulus (right), versus frequency, for the $10 \% w / w \mathrm{BaTiO}_{3}-\mathrm{M}_{4} \mathrm{~A}_{4} \mathrm{PA}_{2}$ nano-composites. 

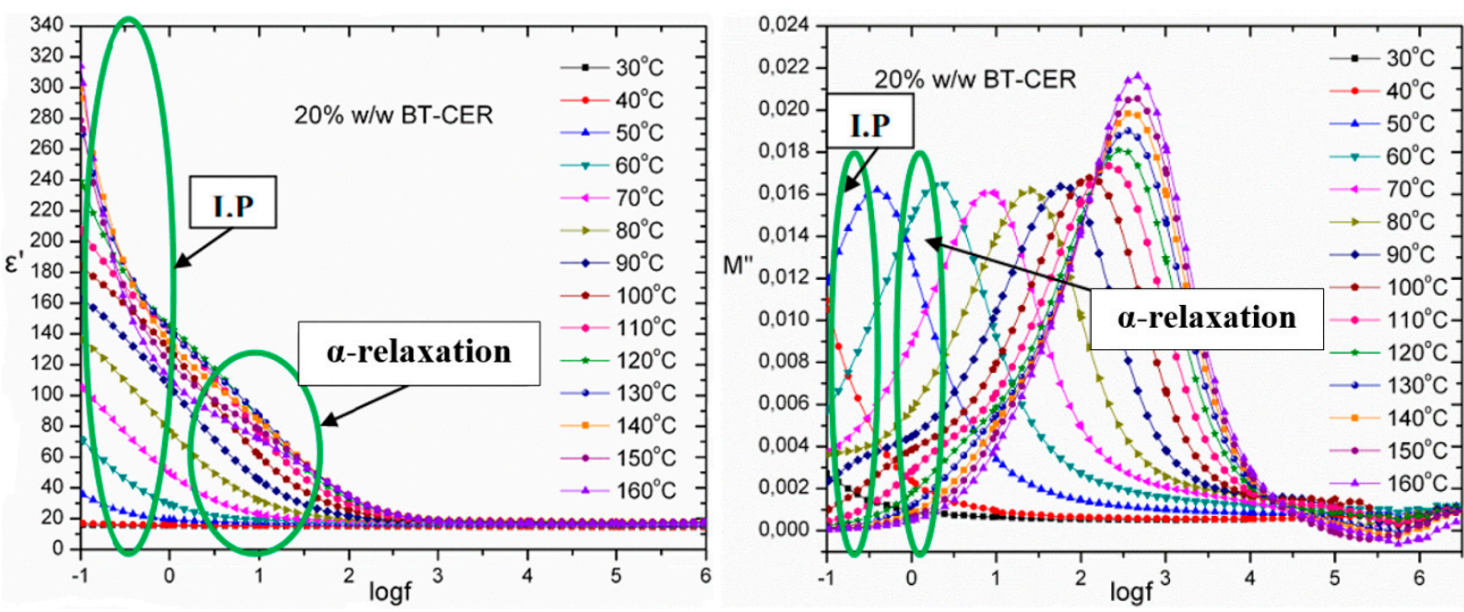

Figure 11. The real part of the dielectric permittivity (left) and the imaginary part of the electric modulus (right), versus frequency, for the $20 \% w / w \mathrm{BaTiO}_{3}-\mathrm{CER}$ nano-composites.

The most important electric property of electric, from all that were examined, was the energy density-a parameter informing us about the magnitude of energy storing capability of each one of the developed composites. The density of energy was calculated via Equation (2):

$$
\langle u\rangle=\frac{1}{2} \cdot \varepsilon_{0} \cdot \varepsilon^{\prime} \cdot\left(\frac{V_{0}}{d}\right)^{2}
$$

where $\varepsilon_{0}$ is the dielectric permittivity of vacuum, $\varepsilon^{\prime}$ is the real part of dielectric permittivity of the corresponding specimen, $\mathrm{V}_{0}$ is the applied voltage amplitude (which in our case was kept constant at $1 \mathrm{~V}$ ), and $\mathrm{d}$ is the specimen's thickness (being equal to $3 \mathrm{~mm}$ ) [1-3,33].

Since the main application of the developed composites was to work as capacitors, the importance of energy density is apparent. Energy density has been calculated for all developed composites versus the whole testing range of frequencies and temperatures. Specific temperature and frequency values were chosen to examine the functionality of the specimens, under certain conditions. These certain conditions consisted of temperature at $30^{\circ} \mathrm{C}$, and frequency at $10^{-1} \mathrm{~Hz}$, values that simulate the functional conditions of a typical environment. In each temperature and frequency for the above-mentioned value, a scan through the whole testing frequency and temperature window took place. Two characteristic graphs of such scanning are shown in Figure 12. Composites with the $\mathrm{M}_{7} \mathrm{~A}_{2} \mathrm{PA}_{1}$ unsaturated polyester as a polymer matrix exhibited the highest energy density values, compared to all others. The energy density acquired its highest values at low frequencies and at high (but not the highest) temperatures. A very crucial observation was that the highest energy density values were not exhibited by the specimens with the highest content of barium titanate. For example, for composites with the $\mathrm{M}_{7} \mathrm{~A}_{2} \mathrm{PA}_{1}$ unsaturated polyester as a polymer matrix, the highest energy density values were shown by the specimen with $10 \% w / w$ content of barium titanate. This was probably due to the specificity of each polymer cured network and the corresponding manner in which the nano-particles were dispersed in it. The shape of the polymer 3D network, defines the mobility of the permanent dipoles, as well as the mobility of the developed dipoles, at the interface of the barium titanate nanoparticles and, therefore, the energy storing capability of each composite material. So, the permittivity did not increase monotonically with the filler content, as expected, because the quality of the achieved dispersion of the nano-particles diminished at high $\mathrm{BaTiO}_{3}$ loadings, in some composite categories. The nano-particles tended to form clusters and agglomerates and the sample's preparation was significantly hampered. The presence of agglomerates, the weak adhesion between particles and matrix, and the possible formation of small voids lead to lower permittivity values for the nanocomposites with a high barium titanate content. Therefore, the corresponding energy density values decreased [1]. The optimum content of barium titanate exhibited the highest energy density values, constituting the most tempting occasions of these 
composites for the developing applications, by also taking into account, the high cost of barium titanates. The specimens with the highest energy density value for each developed composite category are listed in Table 10. It was obvious that in several composite categories, the highest energy density values were not shown by the specimens with the maximum content of barium titanate. Regarding the comparison between the composites, in relation to each polymer matrix, the lowest energy density was exhibited by the specimens with commercial unsaturated polyester resin and commercial epoxy resin as a polymer matrix. Then with slightly higher energy density values, followed the composites with the laboratory novolac resin as a polymer matrix. The highest energy density values were exhibited by the composites with laboratory unsaturated polyesters, as a polymer matrix, with the highest value being exhibited by the specimen with the $10 \% w / w$ BT- $\mathrm{M}_{7} \mathrm{~A}_{2} \mathrm{PA}_{1}$.
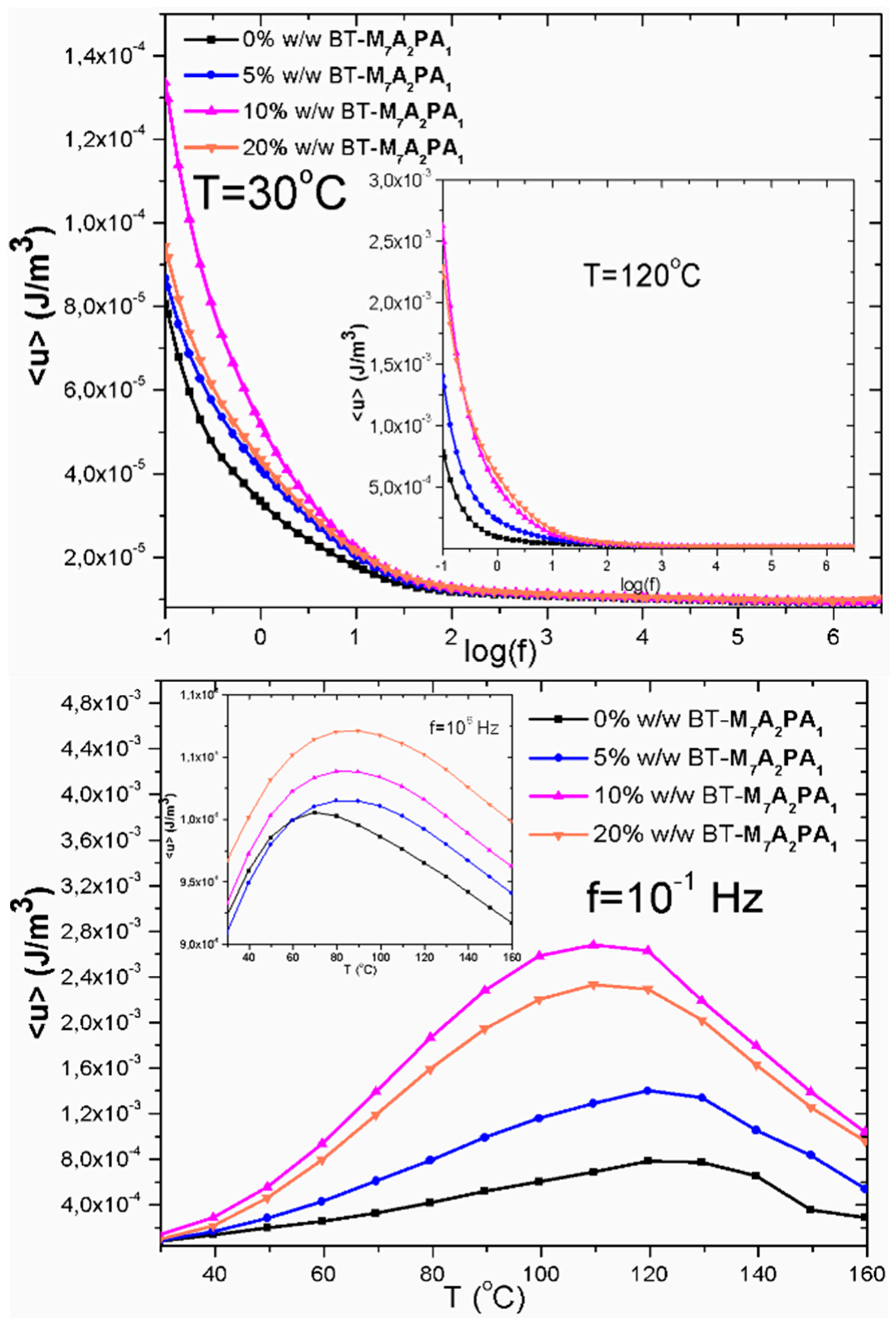

Figure 12. Comparative graphs of energy density versus frequency (up), at $30^{\circ} \mathrm{C}$ and $120^{\circ} \mathrm{C}$ (inset), and versus temperature (down), at $10^{-1} \mathrm{~Hz}$ and $10^{6} \mathrm{~Hz}$ (inset), for the $\mathrm{M}_{7} \mathrm{~A}_{2} \mathrm{PA}_{1}-\mathrm{BT}$ nano- composites. 
Table 10. Maximum energy density values versus frequency (at $30{ }^{\circ} \mathrm{C}$ ) and versus temperature (at $10^{-1} \mathrm{~Hz}$ ), in relation to the composite's content of barium titanate, for all developed categories of composite materials.

\begin{tabular}{|c|c|c|c|c|}
\hline \multirow{2}{*}{ Type of Composite } & \multicolumn{2}{|c|}{$<\mathrm{u}>v s \operatorname{logf}$ Graphs, at $30^{\circ} \mathrm{C}$} & \multicolumn{2}{|c|}{$<u>v s$ Temperature Graphs, at $10^{-1} \mathrm{~Hz}$} \\
\hline & $\%$ w/w $\mathrm{BaTiO}_{3}$ & $<\mathrm{u}\rangle_{\max }\left(\mathrm{J} / \mathrm{m}^{3}\right)$ & $\%$ w/w $\mathrm{BaTiO}_{3}$ & $<\mathrm{u}>_{\max }\left(\mathrm{J} / \mathrm{m}^{3}\right)$ \\
\hline NV-BT & 10 & $1.15 \times 10^{-5}$ & 10 & $7.40 \times 10^{-5}$ \\
\hline CUP-BT & 20 & $470 \times 10^{-6}$ & 5 & $5.25 \times 10^{-4}$ \\
\hline $\mathrm{M}_{1} \mathrm{~A}_{3} \mathrm{PA}_{6}-\mathrm{BT}$ & 20 & $3.50 \times 10^{-5}$ & 20 & $3.60 \times 10^{-4}$ \\
\hline $\mathrm{M}_{3} \mathrm{~A}_{4} \mathrm{PA}_{3}-\mathrm{BT}$ & 20 & $2.30 \times 10^{-5}$ & 20 & $2.80 \times 10^{-4}$ \\
\hline $\mathrm{M}_{4} \mathrm{~A}_{4} \mathrm{PA}_{2}-\mathrm{BT}$ & 10 & $3.62 \times 10^{-5}$ & 10 & $2.00 \times 10^{-4}$ \\
\hline $\mathrm{M}_{7} \mathrm{~A}_{2} \mathrm{PA}_{1}-\mathrm{BT}$ & 10 & $1.35 \times 10^{-4}$ & 10 & $2.80 \cdot 10^{-3}$ \\
\hline $\mathrm{M}_{6} \mathrm{~A}_{4}-\mathrm{BT}$ & 5 & $2.30 \times 10^{-5}$ & 10 & $2.23 \times 10^{-3}$ \\
\hline CER-BT & 20 & $7.75 \times 10^{-6}$ & 20 & $1.50 \times 10^{-4}$ \\
\hline
\end{tabular}

\section{Discussion}

The purpose of the present study was to develop novel polymer nanocomposites by combining the three most important and communal thermosetting polymer matrices with barium titanate nano-particles. The produced systems can be considered to be a new generation of nanocomposites, since they incorporate a distributed network of nanocapacitors. This network can be exploited for energy storage and, thus, a type of nano-device has been defined.

Barium titanate is known for its energy storing capability and, as a result, it is a widely used material for capacitors. Combining this new generation material with the most important thermosetting polymer, as matrices, completely new and innovative materials were developed. First, this research dealt with the synthesis process and kinetic analysis that take place in unsaturated polyesters. In the first part of this research work, a synthesis and kinetic analysis of unsaturated polyesters took place. The steps that followed concerned with the curing process-a compression molding technique-and was carried out via a thermo-compressor. The next stage was to carry out the structural characterization via Scanning Electron Microscopy, assisted with Electron Dispersive X-Ray Spectroscopy, X-Ray Diffraction, and Infrared Spectroscopy, via a Fourier Transformation. The glass transition temperature for all studied nanocomposites was determined via a differential scanning calorimetry. Then, the mechanical properties were studied via the three-point method, in order to calculate the shear and bending strength values for all developed specimens. Finally, the most important study consisted of the Broadband Dielectric Spectroscopy, as dielectric properties hold crucial importance for this kind of composite materials. In particular, the dielectric permittivity, electric modulus, and energy density were determined. Energy density was the most important parameter as it is the measure that quantifies the energy storing capability of a material. It was found that the optimum dielectric performance did not correspond to the maximum filler loading, in all composite types, and the highest values were exhibited by the $10 \% w / w$ $\mathrm{BT}-\mathrm{M}_{7} \mathrm{~A}_{2} \mathrm{PA}_{1}$.

\section{Conclusions}

The above research sums up data and very important conclusion of a multi-annual study. It involves the three most important and common thermosetting polymers as polymers matrices and multi-functional nano-particles of barium titanate. So, there is not one composite better than the other, the only factor that matters its time is the certain application. The application defines the properties that each material must have, so if the in the application the engineer prefers higher energy density then laboratory M7A2PA1 should be the better choice, but if the application needs high mechanical properties in shear and bending strength then commercial epoxy resin composites might be a better option. Summarizing this article presents all the measured properties and the study of their structure in this concise comparative study. 
Author Contributions: Conceptualization, I.A.A.; methodology, L.Z.; software, G.C.P. and L.Z.; validation, I.A.A., G.C.P. and L.Z.; formal analysis, I.A.A.; investigation, I.A.A.; resources, I.A.A.; data curation, I.A.A.; writing—original draft preparation, I.A.A.; writing—review and editing, I.A.A.; visualization, I.A.A., G.C.P., and L.Z.; supervision, G.C.P. and L.Z.; project administration, I.A.A.; funding acquisition, G.C.P. and L.Z.

Funding: This research received no external funding.

Conflicts of Interest: The authors declare no conflict of interest.

\section{References}

1. Asimakopoulos, I.A.; Psarras, G.C.; Zoumpoulakis, L. Barium titanate/polyester resin nanocomposites: Development, structure-properties relationship and energy storage capability. Express Polym. Lett. 2014, 8, 692-707. [CrossRef]

2. Asimakopoulos, I.; Zoumpoulakis, L.; Psarras, G.C. Development and Characterization of a Novolac Resin $/ \mathrm{BaTiO}_{3}$ Nanoparticles Composite System. J. Appl. Polym. Sci. 2012, 125, 3737-3744. [CrossRef]

3. Asimakopoulos, I.A. Development, Characterization and Properties of Polymer Matrix Dielectric Composite Materials. Ph.D. Thesis, National Technical University of Athens, Athens, Greece, 2015.

4. Abraham, R.; Thomas, S.P.; Kuryan, S.; Isac, J.; Varughese, K.T.; Thomas, S. Mechanical properties of ceramic-polymer nanocomposites. Express Polym. Lett. 2009, 3, 177-189. [CrossRef]

5. Smay, J.E.; Cerano, S.; Tuttle, B.A.; Lewis, J.A. Piezo electric properties of 3-X periodic $\mathrm{Pb}\left(\mathrm{Zr}_{\mathrm{x}} \mathrm{Ti}_{1-\mathrm{x}}\right) \mathrm{O}_{3}$-polymer composites. J. Appl. Phys. 2002, 92, 6119-6127. [CrossRef]

6. Pradhan, D.K.; Choudhary, R.N.P.; Samantaray, B.K. Studies of structural, thermal and electrical behavior of polymer nanocomposites electrolytes. Express Polym. Lett. 2008, 2, 630-638. [CrossRef]

7. Simitzis, J. Correlation between the production parameters and the mechanical properties of novolac resins reinforced with carbon fibers. J. Angew. Makromol. Chem. 1989, 165, 21-34. [CrossRef]

8. Braun, D.; Cherdron, H.; Kern, W. Praktikum der Makromolekularen Organischen Chemie; Huthig Verlag: Heidelberg, Germany, 1971.

9. Simitzis, J.; Karagianis, K.; Zoumpoulakis, L. Curing of novolac-Lignocellulosic Composites. Polym. Int. 1995, 38, 183-189. [CrossRef]

10. Simitzis, J.; Karagianis, K.; Zoumpoulakis, L. Influence of biomass on the curing of novolac-composites. Eur. Polym. J. 1996, 32, 857-863. [CrossRef]

11. Asimakopoulos, I.; Psarras, G.C.; Zoumpoulakis, L. Development and Characterization of a Novolac resin $/ \mathrm{BaTiO}_{3}$ nanoparticles composite system. In Proceedings of the 8th Hellenic Polymer Society Symposium (HPOL8), Hersonissos, Crete, Greece, 24-29 October 2010; p. 215.

12. Asimakopoulos, I.A.; Psarras, G.C.; Zoumpoulakis, L. Study on maleic acid/adipic acid/phthalic anhydride/ethylene glycol based unsaturated polyesters, with embedded ferroelectric $\mathrm{BaTiO}_{3}$ nano-particles, composite systems. In Proceedings of the 10th Hellenic Polymer Society Conference (10th HPSC) with International Participation, Patras, Greece, 4-6 December 2014; pp. 296-298.

13. Simitzis, J.; Zoumpoulakis, L.; Soulis, S. Effect of composition and polyesterification catalysts on the optical properties of cured polyesters. Polym. Int. 2002, 51, 297-307. [CrossRef]

14. Simitzis, J.; Zoumpoulakis, L.; Soulis, S. DSC curing study of catatytically synthesized maleic-acid-based unsaturated polyesters. Polym. Int. 2002, 51, 308-318. [CrossRef]

15. Simitzis, J.; Zoumpoulakis, L.; Soulis, S. Review of the research results concerning the synthesis, curing, structure and properties of unsaturated polyesters. Curr. Trends Polym. Sci. 2003, 8, 107-125.

16. Pomakis, I.; Simitzis, I. A new method to control the polyesterification process. Prospects of application in the production plants. Angew. Makromol. Chem. 1981, 99, 145-170. [CrossRef]

17. Asimakopoulos, I.A.; Psarras, G.C.; Zoumpoulakis, L. Mechanical and Dielectric Properties of Barium Titanate/Polyester Nano-Composite Materials. In Proceedings of the 9th International Conference on Nanosciences \& Nanotechnologies (NN12), Thessaloniki, Greece, 3-6 July 2012; p. 215.

18. Asimakopoulos, I.A.; Psarras, G.C.; Zoumpoulakis, L. Mechanical and Dielectric Properties versus Structure: Study of epoxy resin / barium titanate nanocomposites. In Proceedings of the 30th Panhellenic Conference on Solid-State Physics and Materials Science (30th SSPMS), Heraklion, Crete, Greece, 21-24 September 2014; p. 187. 
19. Asimakopoulos, I.A.; Psarras, G.C.; Zoumpoulakis, L. Structure-Properties relationship and Energy Storage capability of Nano-Composite System: Polyester polymer matrix/barium titanate particles. In Proceedings of the 28th Panhellenic Conference on Solid State Physics and Materials Science (28th SSPMS), Patras, Greece, 23-26 September 2012; Available online: http:/ / xxviii.physics.upatras.gr/program.html (accessed on 20 September 2012).

20. Asimakopoulos, I.A.; Psarras, G.C.; Zoumpoulakis, L. Composite Materials Comparative Study: Consisting of three kinds of Thermosetting Polymer Matrices (phenol-formaldehyde, 8 kinds of different composition unsaturated polyesters and epoxy) and Ferroelectric Ceramic Nano-Particles of Barium Titanate. In Proceedings of the 10th Chemical Engineering Panhellenic Conference, Patras, Greece, 4-6 June 2015; p. 49.

21. Orthamann, M. Die Prüfung thermoplastischer Kunstoffe; Carl Hanser Verlag: München, Germany, 1971.

22. Bürger, A. Kohlenstoffasen-Verstärker Polymere und deren Ehernischer, Abbaw bis zu Kohlenstoff-Verbund-Werkstoffen (in German). Ph.D. Thesis, Karlsruhe Institute of Technology, Karlsruhe, Germany, 1973.

23. Skoog, D.A.; Holler, F.J.; Nieman, T.A. Principles in Instrumental Analysis; Kostarakis Publ.: Athens, Greece, 2005.

24. Valavanides, T. Basic Principles in Molecular Spectroscopy and Applications in Organic Chemistrie; Sichrona Themata Publ:: Athens, Greece, 2008.

25. Mc Murry, J. Organic Chemistry; Crete University Publ.: Herakleion, Greek, 2007.

26. Atta, A.M.; Nassar, I.F.; Bedawy, H.M. Unsaturated polyester resins based on rosin maleic anhydride adduct as corrosion protections of steel. React. Funct. Polym. 2007, 67, 617-626. [CrossRef]

27. Abdallh, M.; Bakir, E.; Yousif, E. Study the electrical conductivity of crosslinked polyester doped with different metal salts. J. Saudi Chem. Soc. 2014, 18, 387-391. [CrossRef]

28. Cho, L.L. Identification of textile fiber by Raman microspectroscopy. Forensic Sci. J. 2007, 6, 55-62.

29. Oswal, S.L.; Pandya, A.K. Synthesis and Characterization of Linear Aromatic Polyester-amides from Diacid Chlorides and Aminophenols. Iran. Polym. J. 2004, 13, 205-212.

30. Psarras, G.C.; Manolakaki, E.; Tsangaris, G.M. Dielectric Dispersion and ac Conductivity in-Iron Particles Loaded-Polymer Composites. J. Compo. A 2003, 34, 1187-1198. [CrossRef]

31. Psarras, G.C.; Manolakaki, E.; Tsangaris, G.M. Electrical Relaxations in Polymeric Particulate Composites of Epoxy Resin and Metal Particles. J. Compos. A 2002, 33, 375-384. [CrossRef]

32. Tsangaris, G.M.; Psarras, G.C.; Kouloumbi, N. Electric modulus and interfacial polarization in composite polymeric systems. J. Mater. Sci. 1998, 33, 2027-2037. [CrossRef]

33. Dang, Z.M.; Yuan, J.K.; Zha, J.W.; Zhou, T.; Li, S.T.; Hu, G.H. Fundamentals, processes and applications of high-permittivity polymer-matrix composites. Prog. Mater. Sci. 2012, 57, 660-723. [CrossRef] 